\title{
CONDITIONS FOR SUPERCONVERGENCE OF HDG METHODS FOR STOKES FLOW
}

\author{
BERNARDO COCKBURN AND KE SHI
}

\begin{abstract}
We provide an a priori error analysis of a wide class of finite element methods for the Stokes equations. The methods are based on the velocity gradient-velocity-pressure formulation of the equations and include new and old mixed and hybridizable discontinuous Galerkin methods. We show how to reduce the error analysis to the verification of some properties of an elementwise-defined projection and of the local spaces defining the methods. We also show that the projection of the errors only depends on the approximation properties of the projection. We then provide sufficient conditions for the superconvergence of the projection of the error in the approximate velocity. We give many examples of these methods and show how to systematically construct them from similar methods for the diffusion equation.
\end{abstract}

\section{INTRODUCTION}

In this paper, we propose a projection-based analysis of superconvergent hybridizable discontinuous Galerkin (HDG) methods for the velocity gradient-velocity-pressure formulation of the Stokes equations, namely,

$$
\begin{aligned}
\mathrm{L}-\nabla \boldsymbol{u} & =0 & & \text { on } \Omega, \\
-\nabla \cdot(\nu \mathrm{L})+\nabla p & =\boldsymbol{f} & & \text { on } \Omega, \\
\nabla \cdot \boldsymbol{u} & =0 & & \text { on } \Omega, \\
\boldsymbol{u} & =\boldsymbol{g} & & \text { on } \partial \Omega, \\
\int_{\Omega} p & =0, & &
\end{aligned}
$$

where $\int_{\partial \Omega} \boldsymbol{g} \cdot \boldsymbol{n}=0$. Here $\Omega \subset \mathbb{R}^{n}(n=2,3)$ is a bounded polygonal domain if $n=2$ or a Lipschitz polyhedral domain if $n=3$. We assume that $\nu$ is a constant.

In [7, we introduced a new technique to carry out the a priori error analysis of HDG methods for second order elliptic problems. The technique reduces the error analysis to the verification of some properties of an elementwise-defined projection and of the local spaces defining the methods. It also reduces the study of the convergence properties of the projection of the errors to that of the approximation properties of the projection. It provides sufficient conditions for the superconvergence of the projection of the error in the scalar approximation; as a consequence, a new scalar approximation can be locally computed which converges with the same order. By using this technique, the well-known mixed methods (Raviart-Thomas

Received by the editor August 22, 2011 and, in revised form, August 26, 2011.

2010 Mathematics Subject Classification. Primary 35L65, 65M60, 65N30.

The first author was supported in part by the National Science Foundation (Grant DMS0712955) and by the University of Minnesota Supercomputing Institute. 
methods [16] and their extension by Nédélec [13, Brezzi-Douglas-Marini methods [2], Brezzi-Douglas-Durán-Fortin methods [1]) as well as old [5] and new HDG methods can be analyzed at once. In this paper, we extend this methodology to the Stokes equations of incompressible flow.

To better describe our results, we adapt to our setting the notation used in [7] and in [4]. Let $\mathcal{T}_{h}$ denote the shape-regular, conforming triangulation of $\Omega$, set $\partial \mathcal{T}_{h}:=\left\{\partial K: K \in \mathcal{T}_{h}\right\}$, and let $\mathcal{E}_{h}$ denote the set of all faces $F$ of all simplexes $K \in \mathcal{T}_{h}$. We denote by $\mathcal{F}(K)$ the set of all faces $F$ of $K$.

The methods we consider seek an approximation $\left(\mathrm{L}_{h}, \boldsymbol{u}_{h}, p_{h}, \widehat{\boldsymbol{u}}_{h}\right)$ to the exact solution $\left(\left.\mathrm{L}\right|_{\Omega},\left.\boldsymbol{u}\right|_{\Omega},\left.p\right|_{\Omega},\left.\boldsymbol{u}\right|_{\mathcal{E}_{h}}\right)$ in the finite dimensional space $\mathrm{G}_{h} \times \boldsymbol{V}_{h} \times P_{h} \times \boldsymbol{M}_{h}$ given by

$$
\begin{array}{rll}
\mathrm{G}_{h}=\left\{\mathrm{G} \in \mathrm{L}^{2}\left(\mathcal{T}_{h}\right):\right. & \left.\mathrm{G}\right|_{K} \in \mathrm{G}(K) & \left.\forall K \in \mathcal{T}_{h}\right\}, \\
\boldsymbol{V}_{h}=\left\{\boldsymbol{v} \in \boldsymbol{L}^{2}\left(\mathcal{T}_{h}\right):\right. & \left.\boldsymbol{v}\right|_{K} \in \boldsymbol{V}(K) & \left.\forall K \in \mathcal{T}_{h}\right\}, \\
P_{h}=\left\{q \in L^{2}\left(\mathcal{T}_{h}\right):\right. & \left.q\right|_{K} \in P(K) & \left.\forall K \in \mathcal{T}_{h}\right\}, \\
\boldsymbol{M}_{h}=\left\{\boldsymbol{\mu} \in \boldsymbol{L}^{2}\left(\mathcal{E}_{h}\right):\right. & \left.\boldsymbol{\mu}\right|_{F} \in \boldsymbol{M}(F) & \left.\forall F \in \mathcal{E}_{h}\right\} .
\end{array}
$$

To describe how the approximation is defined, we need to introduce some notation related to integrals on the triangulation $\mathcal{T}_{h}$. Here, we write $(\mathrm{N}, \mathrm{Z})_{\mathcal{T}_{h}}:=$ $\sum_{i, j=1}^{n}\left(\mathrm{~N}_{i, j}, \mathrm{Z}_{i, j}\right)_{\mathcal{T}_{h}},(\boldsymbol{\eta}, \boldsymbol{\zeta})_{\mathcal{T}_{h}}:=\sum_{i=1}^{n}\left(\eta_{i}, \zeta_{i}\right)_{\mathcal{T}_{h}}$, and $(\eta, \zeta)_{\mathcal{T}_{h}}:=\sum_{K \in \mathcal{T}_{h}}(\eta, \zeta)_{K}$, where $(\eta, \zeta)_{D}$ denotes the integral of $\eta \zeta$ over $D \subset \mathbb{R}^{n}$. In a similar way, we write $\langle\boldsymbol{\eta}, \boldsymbol{\zeta}\rangle_{\partial \mathcal{T}_{h}}:=\sum_{i=1}^{n}\left\langle\eta_{i}, \zeta_{i}\right\rangle_{\partial \mathcal{T}_{h}}$ and $\langle\eta, \zeta\rangle_{\partial \mathcal{T}_{h}}:=\sum_{K \in \mathcal{T}_{h}}\langle\eta, \zeta\rangle_{\partial K}$, where $\langle\eta, \zeta\rangle_{D}$ denotes the integral of $\eta \zeta$ over $D \subset \mathbb{R}^{n-1}$.

The approximation $\left(\mathrm{L}_{h}, \boldsymbol{u}_{h}, p_{h}, \widehat{\boldsymbol{u}}_{h}\right)$ can now be defined as the solution of the following equations:

$$
\begin{aligned}
\left(\nu \mathrm{L}_{h}, \mathrm{G}\right)_{\mathcal{T}_{h}}+\left(\boldsymbol{u}_{h}, \nabla \cdot \mathrm{G}\right)_{\mathcal{T}_{h}}-\left\langle\widehat{\boldsymbol{u}}_{h}, \mathrm{G} \boldsymbol{n}\right\rangle_{\partial \mathcal{T}_{h}} & =0 \\
\left(\nu \mathrm{L}_{h}, \nabla \boldsymbol{v}\right)_{\mathcal{T}_{h}}-\left(p_{h}, \nabla \cdot \boldsymbol{v}\right)_{\mathcal{T}_{h}}-\left\langle\nu \widehat{\mathrm{L}}_{h} \boldsymbol{n}-\widehat{p}_{h} \boldsymbol{n}, \boldsymbol{v}\right\rangle_{\partial \mathcal{T}_{h}} & =(\boldsymbol{f}, \boldsymbol{v})_{\mathcal{T}_{h}}, \\
-\left(\boldsymbol{u}_{h}, \nabla q\right)_{\mathcal{T}_{h}}+\left\langle\widehat{\boldsymbol{u}}_{h} \cdot \boldsymbol{n}, q\right\rangle_{\partial \mathcal{T}_{h}} & =0 \\
\left\langle\widehat{\boldsymbol{u}}_{h}, \boldsymbol{\mu}\right\rangle_{\partial \Omega} & =\langle\boldsymbol{g}, \boldsymbol{\mu}\rangle_{\partial \Omega}, \\
\left\langle\nu \widehat{\mathrm{L}}_{h} \boldsymbol{n}-\widehat{p}_{h} \boldsymbol{n}, \boldsymbol{\mu}\right\rangle_{\partial \mathcal{T}_{h} \backslash \partial \Omega} & =0, \\
\left(p_{h}, 1\right)_{\mathcal{T}_{h}} & =0,
\end{aligned}
$$

for all $(\mathrm{G}, \boldsymbol{v}, q, \boldsymbol{\mu}) \in \mathrm{G}_{h} \times \boldsymbol{V}_{h} \times P_{h} \times \boldsymbol{M}_{h}$, where

$$
\nu \widehat{\mathrm{L}}_{h} \boldsymbol{n}-\widehat{p}_{h} \boldsymbol{n}=\nu \mathrm{L}_{h} \boldsymbol{n}-p_{h} \boldsymbol{n}-\boldsymbol{\alpha}\left(\boldsymbol{u}_{h}-\widehat{\boldsymbol{u}}_{h}\right) \quad \text { on } \partial \mathcal{T}_{h} .
$$

Note that, by taking particular choices of the local spaces $\mathrm{G}(K), \boldsymbol{V}(K), P(K)$ and $\boldsymbol{M}(F)$, and of the linear local stabilization operator $\boldsymbol{\alpha}$, different mixed and HDG methods are obtained.

Our main result is that if we can construct an auxiliary projection $\Pi_{h}(\mathrm{~L}, \boldsymbol{u}, p)=$ $\left(\Pi_{G} \mathrm{~L}, \Pi_{V} \boldsymbol{u}, \Pi_{P} p\right)$ satisfying certain orthogonality and approximation conditions, and if the local spaces $\mathrm{G}(K), \boldsymbol{V}(K), P(K)$ and $\boldsymbol{M}(F)$, for $F \in \mathcal{F}(K)$, satisfy some inclusion properties, then the method is well defined and we have the estimates

$$
\begin{aligned}
\left\|\mathrm{L}-\mathrm{L}_{h}\right\|_{\mathcal{T}_{h}} & \leq 2\left\|\mathrm{~L}-\Pi_{G} \mathrm{~L}\right\|_{\mathcal{T}_{h}}, \\
\left\|p-p_{h}\right\|_{\mathcal{T}_{h}} & \leq C_{1}\left(\left\|\Pi_{P} p-p\right\|_{\mathcal{T}_{h}}+\left\|\mathrm{L}-\Pi_{G} \mathrm{~L}\right\|_{\mathcal{T}_{h}}\right), \\
\left\|\Pi_{V} \boldsymbol{u}-\boldsymbol{u}_{h}\right\|_{\mathcal{T}_{h}} & \leq C_{2} h\left\|\mathrm{~L}-\Pi_{G} \mathrm{~L}\right\|_{\mathcal{T}_{h}},
\end{aligned}
$$


where $\|\cdot\|_{\mathcal{T}_{h}}$ denotes the $L^{2}\left(\mathcal{T}_{h}\right)$-norm and the constants $C_{1}, C_{2}$ solely depend on the stabilization operator $\boldsymbol{\alpha}$.

Note that if the error $\Pi_{V} \boldsymbol{u}-\boldsymbol{u}_{h}$ converges to zero faster than the error $\boldsymbol{u}-\boldsymbol{u}_{h}$, this superconvergence property can be advantageously exploited. To do that, we follow [11, 17, 18] and define a new approximation to $\boldsymbol{u}, \boldsymbol{u}_{h}^{*}$, in the space

$$
\boldsymbol{V}_{h}^{*}:=\left\{\boldsymbol{v} \in \boldsymbol{L}^{2}\left(\mathcal{T}_{h}\right):\left.\boldsymbol{v}\right|_{K} \in \boldsymbol{V}^{*}(K) \supset \boldsymbol{P}^{0}(K), K \in \mathcal{T}_{h}\right\},
$$

as follows. On each element $K \in \mathcal{T}_{h}$, the postprocessing $\boldsymbol{u}_{h}^{*}$ is the element of $\boldsymbol{V}^{*}(K)$ such that

$$
\begin{array}{rlrl}
\left(\nabla \boldsymbol{u}_{h}^{*}, \nabla \boldsymbol{v}\right)_{K} & =\left(\mathrm{L}_{h}, \nabla \boldsymbol{v}\right)_{K} & & \forall \boldsymbol{v} \in \boldsymbol{V}^{*}(K), \\
\left(\boldsymbol{u}_{h}^{*}, \boldsymbol{v}\right)_{K}=\left(\boldsymbol{u}_{h}, \boldsymbol{v}\right)_{K} & \forall \in \boldsymbol{P}^{0}(K) .
\end{array}
$$

Here $\boldsymbol{P}^{0}(K)$ denotes the space of vector constant functions on $K$. We are going to show that by properly choosing the spaces $\boldsymbol{V}_{h}^{*}(K), K \in \mathcal{T}_{h}$, we can make $\left\|\boldsymbol{u}_{h}^{*}-\boldsymbol{u}\right\|_{\mathcal{T}_{h}}$ converge as fast as $\left\|\boldsymbol{\Pi}_{V} \boldsymbol{u}-\boldsymbol{u}_{h}\right\|_{\mathcal{T}_{h}}$ to zero.

The a priori error analysis of the HDG method proposed in 4 is now a particular case of our general approach. However, we provide an alternative definition of the auxiliary projection to carry it out. The key property of this new projection is that the definition of $\Pi_{P} p$ is completely decoupled from the definition of $\left(\Pi_{G} \mathrm{~L}, \Pi_{V} \boldsymbol{u}\right)$. This not only considerably simplifies the study of its approximation properties but allows us to see how to systematically construct the HDG methods under consideration from the HDG methods for diffusion considered in [7. To the best of the authors' knowledge, the methods obtained by using this construction seem to be new, except, of course, for the HDG methods on simplexes in 15, 4. It is worth noting that a particular case of the family of methods employing $k$-th order RaviartThomas elements for the approximate velocity gradient is already known. Indeed, the two-dimensional case with $k=0$ is nothing but the mixed method proposed in 9] for triangles and in [10 for rectangles; general, convex quadrilaterals were also considered in [10].

The rest of the paper is organized as follows. In Section 2, we describe the conditions on the auxiliary projection $\Pi_{h}$ and on the local spaces associated with our finite element methods; we then state and discuss the corresponding a priori error estimates. In Section 3, we show how to systematically construct HDG methods with superconvergent velocities from superconvergence HDG methods for diffusion. In Section 4, we provide detailed proofs for the main results. In Section 5, we give a simple proof for the approximation property of the projection mentioned above. We then end with some concluding remarks in Section 6 which include the sketch of the extension of our approach of the HDG methods for linear elasticity recently proposed in [14.

\section{MAin RESUltS}

In this section we show how an a priori error analysis of the HDG methods can be reduced to the verification of a few conditions on the local spaces and on some properties of an associated, auxiliary projection $\Pi_{h}$ defined in an element-byelement fashion. The main idea of our error analysis is to estimate the projection of the errors $\Pi_{h}\left(\mathrm{~L}-\mathrm{L}_{h}, \boldsymbol{u}-\boldsymbol{u}_{h}, p-p_{h}\right)$ and then deduce bounds of the $L^{2}(\Omega)$-norm of the errors $\mathrm{L}-\mathrm{L}_{h}, \boldsymbol{u}-\boldsymbol{u}_{h}, p-p_{h}$ and $\boldsymbol{u}-\boldsymbol{u}^{*}$. 
2.1. Estimate of $\Pi_{G} \mathrm{~L}-\mathrm{L}_{h}$ and $\Pi_{P} p-p_{h}$. Our first result gives an estimate of the projection of the error $\Pi_{G} \mathrm{~L}-\mathrm{L}_{h}$ solely in terms of the approximation error of the projection $\mathrm{L}-\Pi_{G} \mathrm{~L}$. Our second result gives an estimate of the projection of the error $\Pi_{P} p-p_{h}$ in terms of the approximation errors $p-\Pi_{P} p$ and $\mathrm{L}-\Pi_{G} \mathrm{~L}$. To state them, we need to describe our assumptions on the projection $\Pi_{h}$ and on the local finite element spaces $\mathrm{G}(K), \boldsymbol{V}(K), P(K)$ and $\boldsymbol{M}(F)$.

Assumption A:

- Orthogonality properties of $\Pi_{h}$. On each element $K$, there exists a projection $\Pi_{h}(\mathrm{~L}, \boldsymbol{u}, p)=\left(\Pi_{G} \mathrm{~L}, \Pi_{V} \boldsymbol{u}, \Pi_{P} p\right) \in \mathrm{G}(K) \times \boldsymbol{V}(K) \times P(K)$ satisfying the following properties:

(A.1) $\left(\Pi_{G} \mathrm{~L}, \mathrm{G}\right)_{K}=(\mathrm{L}, \mathrm{G})_{K} \quad$ for all $\mathrm{G} \in \nabla \boldsymbol{V}(K)$.

$(A .2)\left(\boldsymbol{\Pi}_{V} \boldsymbol{u}, \boldsymbol{v}\right)_{K}=(\boldsymbol{u}, \boldsymbol{v})_{K} \quad$ for all $\boldsymbol{v} \in \nabla \cdot \mathrm{G}(K)+\nabla P(K)$.

(A.3) $\left(\Pi_{P} p, q\right)_{K}=(p, q)_{K} \quad$ for all $q \in \nabla \cdot \boldsymbol{V}(K)$.

$(A .4)$ For $F \in \mathcal{F}(K)$,

$\left\langle\nu \Pi_{G} \mathrm{~L} \boldsymbol{n}-\Pi_{P} p \boldsymbol{n}-\boldsymbol{\alpha}\left(\boldsymbol{\Pi}_{V} \boldsymbol{u}\right), \boldsymbol{\mu}\right\rangle_{F}=\left\langle\nu \mathrm{L} \boldsymbol{n}-p \boldsymbol{n}-\boldsymbol{\alpha}\left(\boldsymbol{\Pi}_{M} \boldsymbol{u}\right), \boldsymbol{\mu}\right\rangle_{F}$ for all $\boldsymbol{\mu} \in \boldsymbol{M}(F)$.

We also need to assume suitable relations between the traces on the faces $F$ of the local spaces $\mathrm{G}(K), \boldsymbol{V}(K)$ and $P(K)$ with the local space $\boldsymbol{M}(F)$.

- Properties of the traces of the local spaces. For each element $K$, and for any of its face $F$, we assume,

$\left.(A .5) \mathrm{G}(K) \boldsymbol{n}\right|_{F} \subset \boldsymbol{M}(F)$,

(A.6) $\left.\boldsymbol{V}(K)\right|_{F} \subset \boldsymbol{M}(F)$,

(A.7) $\left.P(K) \boldsymbol{n}\right|_{F} \subset \boldsymbol{M}(F)$.

Here, $\left.\mathrm{G}(K) \boldsymbol{n}\right|_{F}$ denotes the space of the traces of normal components of functions of $\mathrm{G}(K)$ on the face $F$ of $K$. Similarly, $\left.\boldsymbol{V}(K)\right|_{F}$ denotes the space of the traces of space $\boldsymbol{V}(K)$ on the face $F$ of $K .\left.P(K) \boldsymbol{n}\right|_{F}$ denotes the traces of functions of $P(K)$ multiplied by unit normal vector $\boldsymbol{n}$ on the face $F$.

Finally, we need a simple assumption on the local stabilization operator $\boldsymbol{\alpha}$ :

- The semi-positivity property of $\boldsymbol{\alpha}$. For each element $K$ and any face $F$,

$(A .8)\langle\boldsymbol{\alpha}(\boldsymbol{\mu}), \boldsymbol{\mu}\rangle_{F} \geq 0$ for all $\boldsymbol{\mu} \in \boldsymbol{M}(F)$.

We are now ready to state our first result. In what follows, we use $\|\cdot\|_{k, D},|\cdot|_{k, D}$ to denote the standard norm and seminorm on any Sobolev space $H^{k}(D)$, respectively. When $k=0$, we omit the index $k$ and simply write $\|\cdot\|_{D}$.

Theorem 2.1. Suppose that the Assumptions A are satisfied. Then we have

$$
\left\|\Pi_{G} \mathrm{~L}-\mathrm{L}_{h}\right\|_{\mathcal{T}_{h}} \leq\left\|\mathrm{L}-\Pi_{G} \mathrm{~L}\right\|_{\mathcal{T}_{h}} .
$$

Note that, we can immediately conclude that $\left\|\mathrm{L}-\mathrm{L}_{h}\right\|_{\mathcal{T}_{h}} \leq 2\left\|\mathrm{~L}-\Pi_{G} \mathrm{~L}\right\|_{\mathcal{T}_{h}}$, and we see that the quality of the approximation $\mathrm{L}_{h}$ depends on the approximation properties of $\Pi_{G} \mathrm{~L}$ only.

Our second result is an estimate for $p-p_{h}$. To state it, we use the following notation for the total average of a function over $\Omega: \bar{p}:=\int_{\Omega} p /|\Omega|$, the following seminorm on $\partial \mathcal{T}_{h}:\|\boldsymbol{\mu}\|_{\boldsymbol{\alpha} / \nu}:=\left\{\sum_{K \in \mathcal{T}_{h}} \frac{1}{\nu}\langle\boldsymbol{\alpha}(\boldsymbol{\mu}), \boldsymbol{\mu}\rangle_{\partial K}\right\}^{\frac{1}{2}}$, and the following auxiliary 
space

$$
\begin{aligned}
& \mathcal{M}_{\boldsymbol{n}, h}^{\perp}:=\left\{\boldsymbol{\mu} \in \boldsymbol{L}^{2}\left(\partial \mathcal{T}_{h}\right): \quad \forall K \in \mathcal{T}_{h}:\left.\quad \boldsymbol{\mu}\right|_{F} \in \boldsymbol{M}(F) \quad \forall F \in \mathcal{F}(K),\right. \\
&\left.\langle\boldsymbol{\mu} \cdot \boldsymbol{n}, q\rangle_{\partial K}=0 \quad \forall q \in P(K): \quad(q, \nabla \cdot \boldsymbol{v})_{K}=0 \quad \forall \boldsymbol{v} \in \boldsymbol{V}(K)\right\} .
\end{aligned}
$$

Theorem 2.2. Suppose that the Assumptions A are satisfied. Then

$$
\left\|\Pi_{P} p-p_{h}\right\|_{\mathcal{T}_{h}} \leq\left|\overline{\Pi_{P} p-p}\right||\Omega|^{\frac{1}{2}}+C C_{p, \alpha / \nu} \nu\left\|\mathrm{L}-\Pi_{G} \mathrm{~L}\right\|_{\mathcal{T}_{h}},
$$

where

$$
C_{p, \alpha / \nu}:=\max \left\{1, \sup _{\boldsymbol{w} \in \boldsymbol{H}_{0}^{1}(\Omega) \backslash\{\mathbf{0}\}} \sup _{\boldsymbol{\mu} \in \mathcal{M}_{n, h}^{\perp} \backslash\{0\}} \frac{\left\langle\boldsymbol{\alpha}(\boldsymbol{\mu}) / \nu, \boldsymbol{P} \boldsymbol{w}-\boldsymbol{\Pi}_{M} \boldsymbol{w}\right\rangle_{\partial \mathcal{T}_{h}}}{\|\boldsymbol{\mu}\|_{\boldsymbol{\alpha} / \nu}\|\boldsymbol{w}\|_{\boldsymbol{H}^{1}(\Omega)}}\right\}
$$

and $\boldsymbol{P}: \boldsymbol{H}^{1}\left(\mathcal{T}_{h}\right) \rightarrow \boldsymbol{V}_{h}$ is any projection such that $(\boldsymbol{P} \boldsymbol{w}-\boldsymbol{w}, \boldsymbol{v})_{\mathcal{T}_{h}}=0$ for all $\boldsymbol{v} \in \boldsymbol{V}_{h}$ such that $\left.\boldsymbol{v}\right|_{K} \in \nabla P(K)$ for all $K \in \mathcal{T}_{h}$.

As a consequence, applying the triangle inequality, we immediately obtain

$$
\left\|p-p_{h}\right\|_{\mathcal{T}_{h}} \leq\left|\overline{\Pi_{P} p-p}\right||\Omega|^{\frac{1}{2}}+\left\|p-\Pi_{P} p\right\|_{\mathcal{T}_{h}}+C C_{p, \alpha / \nu} \nu\left\|\mathrm{L}-\Pi_{G} \mathrm{~L}\right\|_{\mathcal{T}_{h}} .
$$

2.2. Estimate of $\Pi_{V} u-u_{h}$. Next, we provide estimates of $\boldsymbol{u}-\boldsymbol{u}_{h}$ and $\boldsymbol{u}-\widehat{\boldsymbol{u}}$. To state the results, we need to introduce the following dual problem. For any given $\boldsymbol{\theta}$ in $\boldsymbol{L}^{2}(\Omega)$, let $(\mathrm{Z}, \boldsymbol{\sigma}, \eta)$ be the solution of

$$
\begin{aligned}
\mathrm{Z}-\nabla \boldsymbol{\sigma}=0 & \text { on } \Omega, \\
\nabla \cdot(\nu \mathrm{Z})-\nabla \eta=\boldsymbol{\theta} & \text { on } \Omega, \\
-\nabla \cdot \boldsymbol{\sigma}=0 & \text { on } \Omega, \\
\boldsymbol{\sigma}=0 & \text { on } \partial \Omega .
\end{aligned}
$$

We assume that, for some real number $s$, we have the regularity property

$$
\nu\|\mathrm{Z}\|_{\mathrm{H}^{s+1}(\omega)}+\nu\|\boldsymbol{\sigma}\|_{\boldsymbol{H}^{s+2}(\Omega)}+\|\eta\|_{H^{s+1}(\Omega)} \leq C_{r e g}\|\boldsymbol{\theta}\|_{\boldsymbol{H}^{s}(\omega)} .
$$

In the two-dimensional case, the above estimate with $s \leq 0$ follows from the results in 12 when the domain is convex. In the three-dimensional case, the above estimate follows from the results in [8] in the following cases: For any polyhedron with Lipschitz boundary, with $s<1 / 2$; for any convex polyhedron, with $s \leq 0$; and with $s<3 / 2$ if, moreover, all the edges have wedge angles at most $2 \pi / 3$ (a cube, for example).

Moreover, we need a couple of additional assumptions.

\section{Assumption B:}

The first assumption is an approximation property of a projection $\Pi_{h}^{*}(\mathrm{~L}, \boldsymbol{u}, p)=$ $\left(\Pi_{G}^{*} \mathrm{~L}, \Pi_{V}^{*} \boldsymbol{u}, \Pi_{P}^{*} p\right)$ which satisfies the assumptions $(A .1)-(A .4)$ where the local stabilization operator $\boldsymbol{\alpha}(\cdot)$ is replaced by its dual $\boldsymbol{\alpha}^{*}(\cdot)$, that is, by the linear function defined by

$$
\langle\boldsymbol{\alpha}(\boldsymbol{v}), \boldsymbol{w}\rangle_{\partial K}=\left\langle\boldsymbol{v}, \boldsymbol{\alpha}^{*}(\boldsymbol{w})\right\rangle_{\partial K} \quad \text { for all } \boldsymbol{v}, \boldsymbol{w} \in \boldsymbol{L}^{2}(\partial K)
$$

- The approximation property of the projection $\Pi_{h}^{*}$. For each element $K$ and any $(\mathrm{L}, \boldsymbol{u}, p) \in \mathrm{H}^{1}(K) \times \boldsymbol{H}^{2}(K) \times H^{1}(K)$,

(B.1) $\left\|\Pi_{G}^{*} \mathrm{~L}-\mathrm{L}\right\|_{K} \leq C_{\mathrm{app}}^{*} h_{K}\left(|\mathrm{~L}|_{1, K}+|\boldsymbol{u}|_{1, K}+|p|_{1, K}\right)$. 
The second assumption is a condition on the local space $\boldsymbol{V}(K)$.

- The local space $\boldsymbol{V}(K)$ is not too small. For each element $K$, we have that

(B.2) $\mathrm{P}^{0}(K) \subset \nabla \boldsymbol{V}(K)$.

Here $P^{0}(K)$ denotes the space of constant functions on $K$.

We are now ready to state our third result. To state it, we need to introduce the following norm for functions $\boldsymbol{\zeta} \in \boldsymbol{L}^{2}\left(\partial \mathcal{T}_{h}\right):\|\boldsymbol{\zeta}\|_{h}:=\left\{\sum_{K \in \mathcal{T}_{h}} h_{K}\|\boldsymbol{\zeta}\|_{\partial K}^{2}\right\}^{1 / 2}$.

Theorem 2.3. Suppose that the Assumptions $A$ and $B$ are satisfied. Also, suppose that the regularity property (2.3) holds. Then we have

$$
\left\|\boldsymbol{\Pi}_{V} \boldsymbol{u}-\boldsymbol{u}_{h}\right\|_{\mathcal{T}_{h}}+\left\|\boldsymbol{\Pi}_{M} \boldsymbol{u}-\widehat{\boldsymbol{u}}_{h}\right\|_{h} \leq C \nu h\left\|\mathrm{~L}-\Pi_{G} \mathrm{~L}\right\|_{\mathcal{T}_{h}} .
$$

2.3. Estimate of $u-u_{h}^{*}$. Note that if the convergence of $\boldsymbol{u}_{h}$ to $\boldsymbol{\Pi}_{V} \boldsymbol{u}$ is faster than that of $\boldsymbol{u}_{h}$ to $\boldsymbol{u}$, we can take the advantage of this superconvergence result to show that the postprocessing $\boldsymbol{u}_{h}^{*}$ defined by (1.4) converges to $\boldsymbol{u}$ as fast as $\boldsymbol{u}_{h}$ superconverges to $\boldsymbol{\Pi}_{V} \boldsymbol{u}$. To obtain the error estimate, we need the following assumption.

Assumption $C$ :

- The local space $\mathrm{G}(K)$ is not too small. For each element $K$, (C.1) $\boldsymbol{P}^{0}(K) \subset \nabla \cdot \mathrm{G}(K)$.

We can now state our last result.

Theorem 2.4. Suppose that the Assumptions A, B, and $C$ are satisfied. Also, suppose that the regularity property (2.3) holds. Then, we have

$$
\left\|\boldsymbol{u}-\boldsymbol{u}_{h}^{*}\right\|_{\mathcal{T}_{h}} \leq\left\|\boldsymbol{\Pi}_{V} \boldsymbol{u}-\boldsymbol{u}_{h}\right\|_{\mathcal{T}_{h}}+C h\left(\left\|\mathrm{~L}-\mathrm{L}_{h}\right\|_{\mathcal{T}_{h}}+\inf _{\boldsymbol{v} \in \boldsymbol{V}_{h}^{*}}\|\nabla(\boldsymbol{u}-\boldsymbol{v})\|_{\mathcal{T}_{h}}\right) .
$$

\section{A template For the CONSTRUCtion of SUPERCONVERGEnT Methods}

Here, we provide a template which reduces, roughly speaking, the devising of superconvergent HDG methods to a suitable choice of the spaces in each element of the triangulation.

3.1. The choice of the local spaces and the stabilization operator. To construct our superconvergent methods, we pick an arbitrary element $K \in \mathcal{T}_{h}$, and proceed as follows:

Step 1: The local space $\mathrm{G}(K) \times \boldsymbol{V}(K)$. We begin by taking a local space $\mathrm{G}(K) \times \boldsymbol{V}(K)$ such that

$$
\begin{gathered}
\mathrm{P}^{0}(K) \subset \nabla \boldsymbol{V}(K) \subset \mathrm{G}(K), \\
(\nabla \cdot \boldsymbol{V}(K)) \mathrm{Id} \subset \mathrm{G}(K), \\
\boldsymbol{P}^{0}(K) \subset \nabla \cdot \mathrm{G}(K) \subset \boldsymbol{V}(K) .
\end{gathered}
$$


Step 2: The local space $\boldsymbol{M}(F)$. Then, for each face $F$ of the element $K$, we find a space $\boldsymbol{M}(F)$ such that

$$
\begin{aligned}
\left.\mathrm{G}(K) \boldsymbol{n}\right|_{F} & \subset \boldsymbol{M}(F), \\
\left.\boldsymbol{V}(K)\right|_{F} & \subset \boldsymbol{M}(F) .
\end{aligned}
$$

This choice has to be made so that

$$
\sum_{F \in \mathcal{F}(K)} \operatorname{dim} \boldsymbol{M}(F) \leq(\operatorname{dim} \mathrm{G}(K)-\operatorname{dim} \nabla \boldsymbol{V}(K))+(\operatorname{dim} \boldsymbol{V}(K)-\nabla \cdot \mathrm{G}(K)) .
$$

Step 3: The auxiliary local space $\widetilde{\mathrm{G}}(K) \times \widetilde{\boldsymbol{V}}(K)$. Next, we find an auxiliary space $\widetilde{\mathrm{G}}(K) \times \widetilde{\boldsymbol{V}}(K)$ satisfying

$$
\begin{aligned}
\nabla \boldsymbol{V}(K) & \subset \widetilde{\mathrm{G}}(K) \subset \mathrm{G}(K), \\
\nabla \cdot \mathrm{G}(K) & \subset \widetilde{\boldsymbol{V}}(K) \subset \boldsymbol{V}(K),
\end{aligned}
$$

such that, if we set

$$
\begin{array}{ll}
\mathrm{G}^{\perp}(K):=\left\{\mathrm{G} \in \mathrm{G}(K):(\mathrm{G}, \widetilde{\mathrm{G}})_{K}=0\right. & \forall \widetilde{\mathrm{G}}(K)\} \\
\boldsymbol{V}^{\perp}(K):=\left\{\boldsymbol{v} \in \boldsymbol{V}(K):(\boldsymbol{v}, \widetilde{\boldsymbol{v}})_{K}=0\right. & \forall \widetilde{\boldsymbol{V}}(K)\}
\end{array}
$$

we have that

$$
\sum_{F \in \mathcal{F}(K)} \operatorname{dim} \boldsymbol{M}(F)=\operatorname{dim} \mathrm{G}^{\perp}(K)+\operatorname{dim} \boldsymbol{V}^{\perp}(K)
$$

and that

$$
\begin{aligned}
\left\|\mathrm{G}^{\perp}\right\|_{K} \leq C_{\mathrm{G}} h_{K}^{1 / 2}\left\|\mathrm{G}^{\perp} \boldsymbol{n}\right\|_{\partial K_{\mathrm{G}}} & \text { for all } \mathrm{G}^{\perp} \in \mathrm{G}^{\perp}(K), \\
\left\|\boldsymbol{v}^{\perp}\right\|_{K} \leq C_{V} h_{K}^{1 / 2}\left\|\boldsymbol{v}^{\perp} \cdot \boldsymbol{n}\right\|_{\partial K_{V}} & \text { for all } \boldsymbol{v}^{\perp} \in \boldsymbol{V}^{\perp}(K),
\end{aligned}
$$

for some subsets $\partial K_{G}$ and $\partial K_{V}$ of $\mathcal{F}(K)$.

Step 4: The local space $P(K)$. Now, we take the local space $P(K)$ such that

$$
\begin{aligned}
\nabla \cdot \boldsymbol{V}(K) & \subset P(K), \\
P(K) \mathrm{Id} & \subset \mathrm{G}(K) .
\end{aligned}
$$

Step 5: The stabilization operator $\alpha$. Finally, we pick the local stabilization operator $\boldsymbol{\alpha}$ satisfying the semi-positivity condition (A.8) and the following properties:

$$
\begin{array}{rlr}
\langle\boldsymbol{\alpha}(\boldsymbol{\eta}), \boldsymbol{\mu}\rangle_{F} & =\langle\boldsymbol{\eta}, \boldsymbol{\alpha}(\boldsymbol{\mu})\rangle_{F} & \text { for all } \boldsymbol{\eta}, \boldsymbol{\mu} \in \boldsymbol{M}(F), \\
\left\langle\boldsymbol{\alpha}\left(\boldsymbol{v}^{\perp}\right), \boldsymbol{v}^{\perp}\right\rangle_{\partial K} \geq C_{\alpha}\left\|\boldsymbol{v}^{\perp} \cdot \boldsymbol{n}\right\|_{\partial K_{V}}^{2} & \text { for all } \boldsymbol{v}^{\perp} \in \boldsymbol{V}^{\perp}(K) .
\end{array}
$$

3.2. Verification of Assumptions $A, B$ and $C$. We claim that the HDG method determined by the above local spaces and stabilization operator does satisfy $A s$ sumptions $A, B$, and $C$. Let us show that this is indeed the case.

It is easy to see that

- Assumption (A.5) is nothing but condition (3.2a),

- Assumption (A.6) is nothing but condition (3.2b),

- Assumption (A.7) follows from condition (3.8b) and Assumption (A.5),

- Assumption (B.2) is nothing but the first inclusion in condition (3.1a),

- Assumption (C.1) is nothing but the first inclusion in condition (3.1c). 
Note that Assumption (A.8) was supposed to hold in Step 5.

To verify the remaining Assumptions, we must introduce an auxiliary projection $\Pi_{h}$. We define, for any element of $\mathrm{H}^{1}(K) \times \boldsymbol{H}^{1}(K) \times H^{1}(K),(\mathrm{L}, \boldsymbol{u}, p)$, the projection $\Pi_{h}(\mathrm{~L}, \boldsymbol{u}, p):=\left(\Pi_{G} \mathrm{~L}, \Pi_{V} \boldsymbol{u}, \Pi_{P} p\right)$ as the element of $\mathrm{G}(K) \times \boldsymbol{V}(K) \times P(K)$ defined as follows. The pressure component is defined by

$$
\left(\Pi_{P} p, q\right)_{K}=(p, q)_{K} \quad \forall q \in P(K),
$$

whereas the remaining components are defined by

$$
\begin{aligned}
\left(\Pi_{G} \mathrm{~L}, \mathrm{G}\right)_{K} & =(\mathrm{L}, \mathrm{G})_{K} & & \forall \mathrm{G} \in \widetilde{\mathrm{G}}(K), \\
\left(\boldsymbol{\Pi}_{V} \boldsymbol{u}, \boldsymbol{v}\right)_{K}= & (\boldsymbol{u}, \boldsymbol{v})_{K} & & \forall \boldsymbol{v} \in \widetilde{\boldsymbol{V}}(K), \\
\left\langle\nu \Pi_{G} \mathrm{~L} \boldsymbol{n}-\boldsymbol{\alpha}\left(\boldsymbol{\Pi}_{V} \boldsymbol{u}\right), \boldsymbol{\mu}\right\rangle_{F}= & \langle\nu \mathrm{L} \boldsymbol{n}-\boldsymbol{\alpha}(\boldsymbol{u}), \boldsymbol{\mu}\rangle_{F} & & \\
& -\left\langle p-\Pi_{P} p, \boldsymbol{\mu} \cdot \boldsymbol{n}\right\rangle_{F} & & \forall \boldsymbol{\mu} \in \boldsymbol{M}(F),
\end{aligned}
$$

for $F \in \mathcal{F}(K)$.

If this projection were well defined, Assumption (A.1) would follow from the second equation defining the projection, (3.10b), and from the first inclusion in condition (3.4a); Assumption (A.2) would follow from the third equation defining the projection, (3.10c), from the first inclusion in condition (3.4b) and from condition (3.8b); Assumption (A.3) from the first equation defining the projection, (3.10a), and the inclusion condition (3.8a); and Assumption (A.4) from the last equation defining the projection, (3.10d). Thus, it remains to prove that the projection is well defined and that it satisfies Assumption (B.1).

Note that since we are assuming that the stabilization operator $\alpha$ is self-adjoint (see condition (3.9a)), we have that $\Pi_{h}^{*}=\Pi_{h}$. Note also that, by condition (3.6), the system of equations defining the projection $\Pi_{h}$ is square. Hence, it is well defined if and only if, when $(\mathrm{L}, \boldsymbol{u}, p)=(0, \mathbf{0}, 0)$, we have that $\Pi_{h}(\mathrm{~L}, \boldsymbol{u}, p)=(0, \mathbf{0}, 0)$. As a consequence, both the existence of the projection $\Pi_{h}$ as well as Assumption (B.1) follow from an approximation result we state next.

To do that, we need to introduce some notation. We denote by $\left(\boldsymbol{P}_{V}, P_{W}, \boldsymbol{P}_{\widetilde{V}}\right)$ the $L^{2}$-projection into the local space $\boldsymbol{V}(K) \times W(K) \times \tilde{\boldsymbol{V}}(K)$. For any face $F$ of the element $K$, we set

$$
\|\alpha\|_{F}:=\sup _{\mu \in M(F) \backslash\{0\}}\|\alpha(\mu)\|_{F} /\|\mu\|_{F},
$$

and define $\|\alpha\|_{D}:=\max _{F \in D}\|\alpha\|_{F}$ where $D$ is any union of faces of $K$. Finally, we set, for $W^{\perp}(K) \neq\{0\}, R_{W^{\perp}}:=\sup _{w \in W^{\perp}(K) \backslash\{0\}} h_{K}^{1 / 2}\|w\|_{\partial K} /\|w\|_{K}$.

We are now ready to state our result.

Theorem 3.1. We have

$$
\begin{aligned}
& \left\|\mathrm{L}-\Pi_{G} \mathrm{~L}\right\|_{K} \leq\left\|\mathrm{I}_{\mathrm{L}}\right\|_{K}+\mathrm{C}_{1} h_{K}^{\frac{1}{2}}\left\|\nu \mathrm{I}_{\mathrm{L}} \boldsymbol{n}-I_{P} \boldsymbol{n}\right\|_{\partial K_{\mathrm{G}}} \\
& +\mathrm{C}_{2} h_{K}\left\|\left(\boldsymbol{I} \boldsymbol{d}-\boldsymbol{P}_{\widetilde{\boldsymbol{V}}}\right)(\nu \nabla \cdot \mathrm{L}-\nabla p)\right\|_{K}+\mathrm{C}_{3} h_{K}^{\frac{1}{2}}\left\|\boldsymbol{I}_{\boldsymbol{u}}\right\|_{\partial K}, \\
& \left\|\boldsymbol{u}-\boldsymbol{\Pi}_{V} \boldsymbol{u}\right\|_{K} \leq\left\|\boldsymbol{I}_{\boldsymbol{u}}\right\|_{K}+\mathrm{C}_{4} h_{K}^{\frac{1}{2}}\left\|\boldsymbol{I}_{\boldsymbol{u}}\right\|_{\partial K}+\mathrm{C}_{5} h_{K}\left\|\left(\boldsymbol{I} \boldsymbol{d}-\boldsymbol{P}_{\widetilde{\boldsymbol{V}}}\right)(\nu \nabla \cdot \mathrm{L}-\nabla p)\right\|_{K}, \\
& \text { Here } \mathrm{I}_{\mathrm{L}}:=\mathrm{L}-\mathrm{P}_{\mathrm{G}} \mathrm{L}, \boldsymbol{I}_{\boldsymbol{u}}:=\boldsymbol{u}-\boldsymbol{P}_{V} \boldsymbol{u} \text { and } I_{p}:=p-P_{W} p \text {. Moreover, } \mathrm{C}_{1}= \\
& C_{G}, \mathrm{C}_{2}=0, \mathrm{C}_{3}=C_{G} C_{V} R_{V^{\perp}}\|\boldsymbol{\alpha}\|_{\partial K_{\mathrm{G}}} / C_{\alpha}, \mathrm{C}_{4}=\mathrm{C}_{5}=0 \text { whenever } \widetilde{W}(K)=W(K) \text {. }
\end{aligned}
$$


Otherwise,

$$
\begin{aligned}
\mathrm{C}_{1} & =C_{G}, \quad \mathrm{C}_{2}=\frac{C_{G} C_{V}^{2} R_{V^{\perp}}}{C_{\alpha}}, \quad \mathrm{C}_{3}=C_{G}\|\boldsymbol{\alpha}\|_{\partial K_{\mathrm{G}}}\left(1+\frac{C_{V}^{2} R_{V^{\perp}}^{2}\|\boldsymbol{\alpha}\|_{\partial K}}{C_{\alpha}}\right), \\
\mathrm{C}_{4} & =\frac{C_{V}^{2} R_{V^{\perp}}\|\boldsymbol{\alpha}\|_{\partial K}}{C_{\alpha}}, \quad \mathrm{C}_{5}=\frac{C_{V}^{2}}{C_{\alpha}} .
\end{aligned}
$$

This result contains the information of how the choice of local spaces and stabilization operator affects the approximation properties of the projection. It indicates how to choose them to obtain optimal orders of convergence. Let us focus our discussion on the estimate of $\left\|\mathrm{L}-\Pi_{G} \mathrm{~L}\right\|_{K}$ as it is the only relevant one for the convergence properties described in the theorems of Section 2.

Note that if we have that $\partial K_{\mathrm{G}} \cap \partial K_{\boldsymbol{V}}=\emptyset$ and if we take $\boldsymbol{\alpha}$ in such a way that $\|\boldsymbol{\alpha}\|_{\partial K_{\mathrm{G}}}=0$, then $\mathrm{C}_{3}=0$. In this case, the approximation properties of $\Pi_{G}$ are solely controlled by the $L^{2}$-projections $\mathrm{P}_{\mathrm{G}}, \Pi_{P}, \boldsymbol{P}_{\widetilde{\boldsymbol{V}}}$. Hence, we get optimal approximation properties if the $L^{2}$-projection $\Pi_{P}$ converges as fast as $\mathrm{P}_{\mathrm{G}}$ to the identity, and if the convergence order of $\boldsymbol{P}_{\widetilde{\boldsymbol{V}}}$ to the identity is one less than that of $\mathrm{P}_{\mathrm{G}}$.

In the general case, it is enough to take the stabilization operator $\boldsymbol{\alpha}$ such that $\|\boldsymbol{\alpha}\|_{\partial K_{\mathrm{G}}}$ and $\|\boldsymbol{\alpha}\|_{\partial K_{\mathrm{G}}} / C_{\alpha}$ are uniformly bounded to ensure that the constants $\mathrm{C}_{1}, \mathrm{C}_{2}$ and $\mathrm{C}_{3}$ are independent of $\boldsymbol{\alpha}$.

3.3. Proof of the approximation properties of $\Pi_{h}$, Theorem [3.1. To prove the estimates of Theorem 3.1, we follow [4. The idea is to estimate the quantities $\delta_{\mathrm{L}}:=\Pi_{G} \mathrm{~L}-\mathrm{P}_{G} \mathrm{~L}$ and $\boldsymbol{\delta}_{\boldsymbol{u}}:=\boldsymbol{\Pi}_{V} \boldsymbol{u}-\boldsymbol{P}_{V} \boldsymbol{u}$, and then use the triangle inequality to obtain the desired estimates. We proceed in three steps.

Step 1: The equations for $\delta_{\mathrm{L}}$ and $\delta_{u}$. By the equations defining the projection $\Pi_{h},(3.10)$, we have that

$$
\begin{aligned}
\left(\delta_{\mathrm{L}}, \mathrm{G}\right)_{K} & =0 & & \forall \mathrm{G} \in \widetilde{\mathrm{G}}(K), \\
\left(\boldsymbol{\delta}_{\boldsymbol{u}}, \boldsymbol{v}\right)_{K} & =0 & & \forall \boldsymbol{v} \in \widetilde{\boldsymbol{V}}(K), \\
\left\langle\nu \delta_{\mathrm{L}} \boldsymbol{n}-\boldsymbol{\alpha}\left(\boldsymbol{\delta}_{\boldsymbol{u}}\right), \boldsymbol{\mu}\right\rangle_{F} & =\left\langle\nu \mathrm{I}_{\mathrm{L}} \boldsymbol{n}-\boldsymbol{\alpha}\left(\boldsymbol{I}_{\boldsymbol{u}}\right)-I_{p} \boldsymbol{n}, \boldsymbol{\mu}\right\rangle_{F} & & \forall \boldsymbol{\mu} \in \boldsymbol{M}(F),
\end{aligned}
$$

for $F \in \mathcal{F}(K)$.

Step 2: The estimate of $\delta_{u}$. Next, we obtain an estimate of $\boldsymbol{\delta}_{\boldsymbol{u}}$. By the definition of $\boldsymbol{V}^{\perp}(K)$, (3.5b), we see that $\boldsymbol{\delta}_{\boldsymbol{u}} \in \boldsymbol{V}^{\perp}(K)$, by the equation (3.11b). If $\boldsymbol{V}^{\perp}(K)=\{\mathbf{0}\}$, then

$$
\left\|\boldsymbol{\delta}_{\boldsymbol{u}}\right\|_{K}=\mathbf{0}
$$

If $\boldsymbol{V}^{\perp}(K) \neq\{\mathbf{0}\}$, we claim that $\boldsymbol{\delta}_{\boldsymbol{u}}$ is the element of $\boldsymbol{V}^{\perp}(K)$, satisfying

$$
\left\langle\boldsymbol{\alpha}\left(\boldsymbol{\delta}_{\boldsymbol{u}}\right), \boldsymbol{v}\right\rangle_{\partial K}=-\left(\left(\boldsymbol{I d}-\boldsymbol{P}_{\tilde{\boldsymbol{V}}}\right)(\nu \nabla \cdot \mathrm{L}-\nabla p), \boldsymbol{v}\right)_{K}+\left\langle\boldsymbol{\alpha}\left(\boldsymbol{I}_{\boldsymbol{u}}\right), \boldsymbol{v}\right\rangle_{\partial K}
$$

for all $\boldsymbol{v} \in \boldsymbol{V}^{\perp}(K)$.

Taking $\boldsymbol{v}:=\boldsymbol{\delta}_{\boldsymbol{u}}$ and applying the Cauchy-Schwarz inequality, we get

$$
\begin{aligned}
\left\langle\boldsymbol{\alpha}\left(\boldsymbol{\delta}_{\boldsymbol{u}}\right), \boldsymbol{\delta}_{\boldsymbol{u}}\right\rangle_{\partial K} \leq & \left\|\left(\boldsymbol{I} \boldsymbol{d}-\boldsymbol{P}_{\widetilde{\boldsymbol{V}}}\right)(\nu \nabla \cdot \mathrm{L}-\nabla p)\right\|_{K}\left\|\boldsymbol{\delta}_{\boldsymbol{u}}\right\|_{K}+\left\|\boldsymbol{\alpha}\left(\boldsymbol{I}_{\boldsymbol{u}}\right)\right\|_{\partial K}\left\|\boldsymbol{\delta}_{\boldsymbol{u}}\right\|_{\partial K} \\
\leq & C_{V} h_{K}^{1 / 2}\left\|\left(\boldsymbol{I} \boldsymbol{d}-\boldsymbol{P}_{\widetilde{\boldsymbol{V}}}\right)(\nu \nabla \cdot \mathrm{L}-\nabla p)\right\|_{K}\left\|\boldsymbol{\delta}_{\boldsymbol{u}} \cdot \boldsymbol{n}\right\|_{\partial K_{V}} \\
& +C_{V} R_{V^{\perp}}\|\boldsymbol{\alpha}\|_{\partial K}\left\|\boldsymbol{I}_{\boldsymbol{u}}\right\|_{\partial K}\left\|\boldsymbol{\delta}_{\boldsymbol{u}} \cdot \boldsymbol{n}\right\|_{\partial K_{V}},
\end{aligned}
$$


by the condition (3.7b). By the condition on the stabilization operator, (3.9b),

$$
\left\|\boldsymbol{\delta}_{\boldsymbol{u}} \cdot \boldsymbol{n}\right\|_{\partial K_{V}} \leq \frac{C_{V}}{C_{\alpha}}\left(h_{K}^{1 / 2}\left\|\left(\boldsymbol{I} \boldsymbol{d}-\boldsymbol{P}_{\widetilde{\boldsymbol{V}}}\right)(\nu \nabla \cdot \mathrm{L}-\nabla p)\right\|_{K}+R_{V^{\perp}}\|\boldsymbol{\alpha}\|_{\partial K}\left\|\boldsymbol{I}_{\boldsymbol{u}}\right\|_{\partial K}\right) \text {. }
$$

Finally, using once again condition (3.7b), we get that

$$
\left\|\boldsymbol{\delta}_{\boldsymbol{u}}\right\|_{K} \leq \frac{C_{V}^{2}}{C_{\alpha}}\left(h_{K}\left\|\left(\boldsymbol{I} \boldsymbol{d}-\boldsymbol{P}_{\widetilde{\boldsymbol{V}}}\right)(\nu \nabla \cdot \mathrm{L}-\nabla p)\right\|_{K}+h_{K}^{1 / 2} R_{V^{\perp}}\|\boldsymbol{\alpha}\|_{\partial K}\left\|\boldsymbol{I}_{\boldsymbol{u}}\right\|_{\partial K}\right) .
$$

It remains to prove the claim. By the equation (3.11c), we have that

$$
\left\langle\nu \delta_{\mathrm{L}} \boldsymbol{n}-\boldsymbol{\alpha}\left(\boldsymbol{\delta}_{\boldsymbol{u}}\right), \boldsymbol{v}\right\rangle_{\partial K}=\left\langle\nu \mathrm{I}_{\mathrm{L}} \boldsymbol{n}-\boldsymbol{\alpha}\left(\boldsymbol{I}_{\boldsymbol{u}}\right)-I_{p} \boldsymbol{n}, \boldsymbol{v}\right\rangle_{\partial K} \quad \forall \boldsymbol{v} \in \boldsymbol{V}^{\perp}(F),
$$

because $\left.\boldsymbol{v}\right|_{F} \in \boldsymbol{M}(F)$ by the inclusion condition (3.2b).

But

$$
\left\langle\nu \delta_{\mathrm{L}} \boldsymbol{n}, \boldsymbol{v}\right\rangle_{\partial K}=\nu\left(\nabla \cdot \delta_{\mathrm{L}}, \boldsymbol{v}\right)_{K}+\nu\left(\delta_{\mathrm{L}}, \nabla \boldsymbol{v}\right)_{K}=0 .
$$

Indeed, we have that $\left(\nabla \cdot \delta_{\mathrm{L}}, \boldsymbol{v}\right)_{K}=0$ by the first inclusion in condition (3.4b) and the fact that $\boldsymbol{v} \in \boldsymbol{V}^{\perp}(K)$, and we have that $\left(\delta_{\mathrm{L}}, \nabla \boldsymbol{v}\right)_{K}=0$ by equation (3.11a) and the first inclusion in condition (3.4a).

Similarly,

$$
\begin{aligned}
\left\langle\nu \mathrm{I}_{\mathrm{L}} \boldsymbol{n}-I_{p} \boldsymbol{n}, \boldsymbol{v}\right\rangle_{\partial K} & =\left(\nu \nabla \cdot \mathrm{I}_{\mathrm{L}}-\nabla I_{p}, \boldsymbol{v}\right)_{K}+\left(\nu \mathrm{I}_{\mathrm{L}}, \nabla \boldsymbol{v}\right)_{K}-\left(I_{p}, \nabla \cdot \boldsymbol{v}\right)_{K} \\
& =\left(\left(\boldsymbol{I} \boldsymbol{d}-\boldsymbol{P}_{\tilde{\boldsymbol{V}}}\right)(\nu \nabla \cdot \mathrm{L}-\nabla p), \boldsymbol{v}\right)_{K} .
\end{aligned}
$$

Indeed, $\left(\nu \nabla \cdot \mathrm{I}_{\mathrm{L}}-\nabla I_{p}, \boldsymbol{v}\right)_{K}=(\nu \nabla \cdot \mathrm{L}-\nabla p, \boldsymbol{v})_{K}=\left(\left(\boldsymbol{I} \boldsymbol{d}-\boldsymbol{P}_{\widetilde{\boldsymbol{V}}}\right)(\nu \nabla \cdot \mathrm{L}-\nabla p), \boldsymbol{v}\right)_{K}$ by the first inclusion in condition (3.4b), the inclusion condition (3.8a and the fact that $\boldsymbol{v} \in \boldsymbol{V}^{\perp}(K)$. Moreover, $\left(\nu \mathrm{I}_{\mathrm{L}}, \nabla \boldsymbol{v}\right)_{K}=0$ by the first inclusion in condition (3.4a) and the definition of $\mathrm{I}_{\mathrm{L}}$, and $\left(I_{p}, \nabla \cdot \boldsymbol{v}\right)_{K}=0$ by the inclusion condition (3.8a) and the definition of $I_{p}$. This proves the claim.

Step 3: The estimate of $\delta_{\mathrm{L}}$. Finally, let us estimate $\delta_{\mathrm{L}}$. By the definition of $\mathrm{G}^{\perp}(K)$, (3.5b), we see that $\delta_{\mathrm{L}} \in \mathrm{G}^{\perp}(K)$, by the equation (3.11a). By the condition (3.7a), this implies that

$$
\left\|\delta_{\mathrm{L}}\right\|_{K} \leq C_{\mathrm{G}} h_{K}^{1 / 2}\left\|\delta_{\mathrm{L}} \boldsymbol{n}\right\|_{\partial K_{\mathrm{G}}},
$$

and by equation (3.11c), that

$$
\left\|\delta_{\mathrm{L}}\right\|_{K} \leq C_{\mathrm{G}} h_{K}^{1 / 2}\left(\left\|\nu \mathrm{I}_{\mathrm{G}} \boldsymbol{n}-I_{p} \boldsymbol{n}\right\|_{\partial K_{\mathrm{G}}}+\left\|\boldsymbol{\alpha}\left(\boldsymbol{\delta}_{\boldsymbol{u}}\right)\right\|_{\partial K_{\mathrm{G}}}+\left\|\boldsymbol{\alpha}\left(\boldsymbol{I}_{\boldsymbol{u}}\right)\right\|_{\partial K_{\mathrm{G}}}\right) .
$$

If $\tilde{\boldsymbol{V}}(K)=\boldsymbol{V}(K), \boldsymbol{\delta}_{\boldsymbol{u}}=\mathbf{0}$, and we get that

$$
\left\|\delta_{\mathrm{L}}\right\|_{K} \leq C_{\mathrm{G}} h_{K}^{1 / 2}\left(\left\|\nu \mathrm{I}_{\mathrm{G}} \boldsymbol{n}-I_{p} \boldsymbol{n}\right\|_{\partial K_{\mathrm{G}}}+\|\boldsymbol{\alpha}\|_{\partial K_{\mathrm{G}}}\left\|\boldsymbol{I}_{\boldsymbol{u}}\right\|_{\partial K_{\mathrm{G}}}\right) .
$$

If $\tilde{\boldsymbol{V}}(K) \neq \boldsymbol{V}(K)$, then

$$
\left\|\delta_{\mathrm{L}}\right\|_{K} \leq C_{\mathrm{G}} h_{K}^{1 / 2}\left(\left\|\nu \mathrm{I}_{\mathrm{G}} \boldsymbol{n}-I_{p} \boldsymbol{n}\right\|_{\partial K_{\mathrm{G}}}+\|\boldsymbol{\alpha}\|_{\partial K_{\mathrm{G}}}\left(C_{V} R_{V^{\perp}}\left\|\boldsymbol{\delta}_{\boldsymbol{u}}\right\|_{\partial K_{\mathrm{G}}}+\left\|\boldsymbol{I}_{\boldsymbol{u}}\right\|_{\partial K_{\mathrm{G}}}\right)\right),
$$

by condition (3.7b). This completes the proof of Theorem 3.1

\section{EXAMPLES OF SUPERCONVERGENT METHODS}

In this section, we give examples of superconvergent HDG methods. We show how to construct them from similar methods for diffusion problems. 
4.1. Using the superconvergent methods for diffusion. Let us begin by considering the HDG method for diffusion upon which we are going to construct HDG methods for Stokes satisfying Assumptions $A, B$ and $C$.

Thus, following [7, we assume that we have local spaces

$$
\boldsymbol{V}^{\mathrm{D}}(K), \quad W^{\mathrm{D}}(K) \text { and } M^{\mathrm{D}}(F),
$$

such that

$$
\begin{aligned}
\left.\boldsymbol{V}^{\mathrm{D}}(K) \cdot \boldsymbol{n}\right|_{F} & \subset M^{\mathrm{D}}(F), \\
\left.W^{\mathrm{D}}(K)\right|_{F} & \subset M^{\mathrm{D}}(F) .
\end{aligned}
$$

such that, if we set

$$
\begin{array}{ccc}
\boldsymbol{V}^{\perp, \mathrm{D}}(K):=\left\{\boldsymbol{v} \in \boldsymbol{V}^{\mathrm{D}}(K):(\boldsymbol{v}, \widetilde{\boldsymbol{v}})_{K}=0\right. & \left.\forall \widetilde{\boldsymbol{v}} \in \widetilde{\boldsymbol{V}}^{\mathrm{D}}(K)\right\}, \\
W^{\perp, \mathrm{D}}(K):=\left\{w \in W^{\mathrm{D}}(K):(w, \widetilde{w})_{K}=0\right. & \left.\forall \widetilde{w} \in \widetilde{W}^{\mathrm{D}}(K)\right\},
\end{array}
$$

we have that

$$
\sum_{F \in \mathcal{F}(K)} \operatorname{dim} M^{\mathrm{D}}(F)=\operatorname{dim} \boldsymbol{V}^{\perp, \mathrm{D}}(K)+\operatorname{dim} W^{\perp, \mathrm{D}}(K),
$$

and that

$$
\begin{aligned}
\left\|\boldsymbol{v}^{\perp}\right\|_{K} \leq C_{V} h_{K}^{1 / 2}\left\|\boldsymbol{v}^{\perp} \cdot \boldsymbol{n}\right\|_{\partial K_{V}} & \text { for all } \boldsymbol{v}^{\perp} \in \boldsymbol{V}^{\perp, \mathrm{D}}(K), \\
\left\|w^{\perp}\right\|_{K} \leq C_{W} h_{K}^{1 / 2}\left\|w^{\perp}\right\|_{\partial K_{W}} & \text { for all } w^{\perp} \in W^{\perp, \mathrm{D}}(K),
\end{aligned}
$$

for some subsets $\partial K_{V}$ and $\partial K_{W}$ of $\mathcal{F}(K)$.

We also assume that we have a stabilization function $\alpha^{\mathrm{D}}$ satisfying the following properties:

$$
\begin{gathered}
\left\langle\alpha^{\mathrm{D}}(\eta), \mu\right\rangle_{F}=\left\langle\eta, \alpha^{\mathrm{D}}(\mu)\right\rangle_{F} \quad \text { for all } \eta, \mu \in M^{\mathrm{D}}(F), \\
\left\langle\alpha^{\mathrm{D}}\left(w^{\perp}\right), w^{\perp}\right\rangle_{\partial K} \geq C_{\alpha}\left\|w^{\perp}\right\|_{\partial K_{W}}^{2} \text { for all } w^{\perp} \in W^{\perp, \mathrm{D}}(K) .
\end{gathered}
$$

We can now define the HDG methods satisfying Assumptions $A, B$ and $C$. To do that, we introduce some notation. In what follows, we denote by $\mathrm{G}_{i}(K)$ the space of all the $i$-th rows of functions in $\mathrm{G}(K)$, and by $\boldsymbol{V}_{i}(K)$ and $\boldsymbol{M}_{i}(F)$ the space of the $i$-th component of functions in $\boldsymbol{V}(K)$ and $\boldsymbol{M}(F)$, respectively, for $i=1, \ldots, d$.

We construct the wanted HDG method by taking its local spaces as

$$
\mathrm{G}_{i}(K):=\boldsymbol{V}^{\mathrm{D}}(K), \quad \boldsymbol{V}_{i}(K):=W^{\mathrm{D}}(K), \quad \boldsymbol{M}_{i}(F):=M^{\mathrm{D}}(F),
$$

for $i=1, \ldots, d$, and by taking the stabilization function as

$$
\boldsymbol{\alpha}_{i}(\boldsymbol{a}):=\alpha^{\mathrm{D}}\left(a_{i}\right),
$$

also for $i=1, \ldots, d$. The choice of the space for the pressure $P(K)$ is more delicate. We have the following result. 
Theorem 4.1. Let $P(K)$ be such that

$$
\sum_{j=1}^{d} \partial_{j} W^{D}(K) \subset P(K) \subset \bigcap_{j=1}^{d}\left\{v_{j}: \boldsymbol{v} \in \boldsymbol{V}^{D}(K): v_{i}=0 \text { for } i \neq j\right\} .
$$

Then the corresponding HDG method satisfies Assumptions A, B and C.

Proof. It suffices to show that the spaces we define above satisfy all the conditions in the template in Section 3.1. In the definition of $\mathrm{G}(K), \boldsymbol{V}(K)$ we can see that the conditions (3.1a), (3.1c), (3.2), (3.4) -(3.7) and (3.9) are nothing but the vector version of the conditions (4.1)-(4.6). We only need to verify the spaces also satisfy (3.1b), 3.8).

The assumption $\sum_{j=1}^{d} \partial_{j} W^{\mathrm{D}}(K) \subset P(K)$ implies that $\nabla \cdot \boldsymbol{V}(K) \subset P(K)$ and the assumption $P(K) \subset \bigcap_{j=1}^{d}\left\{v_{j}: \boldsymbol{v} \in \boldsymbol{V}^{\mathrm{D}}(K): v_{i}=0\right.$ for $\left.i \neq j\right\}$ such that $P(K) I d \subset \mathrm{G}(K)$. Hence condition (3.8) holds and so

$$
(\nabla \cdot \boldsymbol{V}(K)) I d \subset P(K) I d \subset \mathrm{G}(K) .
$$

In other words, condition $3.1 \mathrm{~b}$ is verified.

4.2. Examples. We now use this construction to devise superconvergent HDG methods for Stokes by using the superconvergent HDG methods for diffusion described in detail in [7].

In the Tables 1 to 4 , we display the orders of convergence for mixed methods and HDG methods using different elements $K$. We only show the space $P(K)$ since the other spaces are given in [7. Note that all the methods achieve optimal orders for $\mathrm{L}_{h}, p_{h}$ and that superconvergence takes place for the projection of the error in $\boldsymbol{u}$. For $k=0$ and two dimensions, the method listed in Table 1 as $\mathbf{R T}_{k}$ is the mixed method proposed in [9], and that the method listed in Table 3 as $\mathbf{R T}_{[k]}$ is the mixed method proposed in [10]; as pointed out in the Introduction, general, convex quadrilaterals were also considered therein.

TABLE 1. Methods for which $k \geq 1$

$K$ simplex

\begin{tabular}{clccc}
\hline $\begin{array}{c}\text { method } \\
\text { for diffusion }\end{array}$ & $P(K)$ & $\left\|\mathrm{G}-\mathrm{G}_{h}\right\|_{\mathcal{T}_{h}}$ & $\left\|p-p_{h}\right\|_{\mathcal{T}_{h}}$ & $\left\|\boldsymbol{u}-\boldsymbol{u}_{h}^{\star}\right\|_{\mathcal{T}_{h}}$ \\
\hline \hline $\mathbf{B D F M}_{k+1}$ & $P^{k}(K)$ & $k+1$ & $k+1$ & $k+2$ \\
$\mathbf{R T}_{k}$ & $P^{k}(K)$ & $k+1$ & $k+1$ & $k+2$ \\
$\mathbf{H D G}_{k}$ & $P^{k}(K)$ & $k+1$ & $k+1$ & $k+2$ \\
$\mathbf{B D M}_{k}$ & $P^{k}(K)$ & $k+1$ & $k+1$ & $k+2$ \\
$k \geq 2$ & & & & \\
\hline
\end{tabular}


TABLE 2. Methods for which $\boldsymbol{M}(F)=\boldsymbol{P}_{k}(F)$ and $k \geq 1$

$K$ square

\begin{tabular}{|c|c|c|c|c|}
\hline $\begin{array}{c}\text { method } \\
\text { for diffusion }\end{array}$ & $P(K)$ & $\left\|\mathrm{G}-\mathrm{G}_{h}\right\|_{\mathcal{T}_{h}}$ & $\left\|p-p_{h}\right\|_{\mathcal{T}_{h}}$ & $\left\|\boldsymbol{u}-\boldsymbol{u}_{h}^{\star}\right\|_{\mathcal{T}_{h}}$ \\
\hline $\mathbf{B D F M}_{[k+1]}$ & $P^{k}(K)$ & $k+1$ & $k+1$ & $k+2$ \\
\hline $\mathbf{H D G}_{[k]}^{P}$ & $P^{k}(K)$ & $k+1$ & $k+1$ & $k+2$ \\
\hline $\mathbf{B D M}_{k \geq 2}[k]$ & $P^{k}(K)$ & $k+1$ & $k+1$ & $k+2$ \\
\hline \multicolumn{5}{|c|}{$K$ cube } \\
\hline $\begin{array}{c}\text { method } \\
\text { for diffusion }\end{array}$ & $P(K)$ & $\left\|\mathrm{G}-\mathrm{G}_{h}\right\|_{\mathcal{T}_{h}}$ & $\left\|p-p_{h}\right\|_{\mathcal{T}_{h}}$ & $\left\|\boldsymbol{u}-\boldsymbol{u}_{h}^{\star}\right\|_{\mathcal{T}_{h}}$ \\
\hline $\mathbf{B D F M}_{[k+1]}$ & $\overline{P^{k}(K)}$ & $\overline{k+1}$ & $\overline{k+1}$ & $\overline{k e+2}$ \\
\hline $\mathbf{H D G}_{[k]}^{P}$ & $P^{k}(K)$ & $k+1$ & $k+1$ & $k+2$ \\
\hline$\underset{k \geq 2}{\mathbf{B D M}_{[k]}^{\left[n_{]}\right.}}$ & $P^{k}(K)$ & $k+1$ & $k+1$ & $k+2$ \\
\hline
\end{tabular}

TABLE 3. Methods for which $\boldsymbol{M}(F)=\boldsymbol{Q}_{k}(F)$ and $k \geq 1$

$K$ square

\begin{tabular}{ccccc}
\hline method & $P(K)$ & $\left\|\mathrm{G}-\mathrm{G}_{h}\right\|_{\mathcal{T}_{h}}$ & $\left\|p-p_{h}\right\|_{\mathcal{T}_{h}}$ & $\left\|\boldsymbol{u}-\boldsymbol{u}_{h}^{\star}\right\|_{\mathcal{T}_{h}}$ \\
\hline \hline $\mathbf{R T}_{[k]}$ & $Q^{k}(K)$ & $k+1$ & $k+1$ & $k+2$ \\
$\mathbf{T N T}_{[k]}$ & $Q^{k}(K)$ & $k+1$ & $k+1$ & $k+2$ \\
$\mathbf{H D G}_{[k]}^{Q}$ & $Q^{k}(K)$ & $k+1$ & $k+1$ & $k+2$ \\
\hline \multicolumn{5}{c}{$K$ cube } \\
\hline $\operatorname{method}^{5} P(K)$ & $\left\|\mathrm{G}-\mathrm{G}_{h}\right\|_{\mathcal{T}_{h}}$ & $\left\|p-p_{h}\right\|_{\mathcal{T}_{h}}$ & $\left\|\boldsymbol{u}-\boldsymbol{u}_{h}^{\star}\right\|_{\mathcal{T}_{h}}$ \\
\hline $\mathbf{R T}_{[k]}$ & $Q^{k}(K)$ & $k+1$ & $k+1$ & $k+2$ \\
$\mathbf{T N T}_{[k]}$ & $Q^{k}(K)$ & $k+1$ & $k+1$ & $k+2$ \\
$\mathbf{H D G}_{[k]}^{Q}$ & $Q^{k}(K)$ & $k+1$ & $k+1$ & $k+2$ \\
\hline
\end{tabular}

TABLE 4. Methods for which $k \geq 1$

$K$ prism

\begin{tabular}{ccccc}
\hline $\begin{array}{c}\text { method } \\
\text { for diffusion }\end{array}$ & $P(K)$ & $\left\|\mathrm{G}-\mathrm{G}_{h}\right\|_{\mathcal{T}_{h}}$ & $\left\|p-p_{h}\right\|_{\mathcal{T}_{h}}$ & $\left\|\boldsymbol{u}-\boldsymbol{u}_{h}^{\star}\right\|_{\mathcal{T}_{h}}$ \\
\hline \hline $\mathbf{B D F M}_{<k+1>}$ & $P^{k}(K)$ & $k+1$ & $k+1$ & $k+2$ \\
$\mathbf{R T}_{<k>}$ & $P^{k \mid k}(K)$ & $k+1$ & $k+1$ & $k+2$ \\
$\mathbf{H D G}_{<k>}$ & $P^{k}(K)$ & $k+1$ & $k+1$ & $k+2$ \\
\hline
\end{tabular}


5. Proofs of the estimates of the projection of the errors

In this section we provide detailed proofs for our a priori error estimates. The main idea is to work with the following projection of the errors:

$$
\begin{aligned}
\mathrm{E}_{\mathrm{L}} & :=\Pi_{G} \mathrm{~L}-\mathrm{L}_{h}, \\
\boldsymbol{e}_{u} & :=\boldsymbol{\Pi}_{V} \boldsymbol{u}-\boldsymbol{u}_{h}, \\
e_{p} & :=\Pi_{P} p-p_{h}, \\
\boldsymbol{e}_{\widehat{u}} & :=\boldsymbol{\Pi}_{M} \boldsymbol{u}-\widehat{\boldsymbol{u}}_{h} .
\end{aligned}
$$

We begin by obtaining the equations satisfied by these projections. We then mimic the argument in [4, 6] to obtain the estimates of $\mathrm{E}_{L}, e_{p}, \boldsymbol{e}_{u}$ and, finally, $\boldsymbol{u}-\boldsymbol{u}_{h}^{*}$.

Step 1: The error equations. We begin by obtaining the equations satisfied by the projection of the errors.

\section{Lemma 5.1.}

$$
\begin{aligned}
& \left(\mathrm{E}_{\mathrm{L}}, \mathrm{G}\right)_{\mathcal{T}_{h}}+\left(\boldsymbol{e}_{u}, \nabla \cdot \mathrm{G}\right)_{\mathcal{T}_{h}}-\left\langle\boldsymbol{e}_{\widehat{u}}, \mathrm{G} \boldsymbol{n}\right\rangle_{\partial \mathcal{T}_{h}}=\left(\Pi_{G} \mathrm{~L}-\mathrm{L}, \mathrm{G}\right)_{\mathcal{T}_{h}}, \\
& -\left(\nabla \cdot\left(\nu \mathrm{E}_{\mathrm{L}}\right), \boldsymbol{v}\right)_{\mathcal{T}_{h}}+\left(\nabla e_{p}, \boldsymbol{v}\right)_{\mathcal{T}_{h}}+\left\langle\boldsymbol{\alpha}\left(\boldsymbol{e}_{u}-\boldsymbol{e}_{\widehat{u}}\right), \boldsymbol{v}\right\rangle_{\partial \mathcal{T}_{h}}=0, \\
& -\left(\boldsymbol{e}_{u}, \nabla q\right)_{\mathcal{T}_{h}}+\left\langle\boldsymbol{e}_{\widehat{u}}, q \boldsymbol{n}\right\rangle_{\partial \mathcal{T}_{h}}=0 \\
& \left\langle\boldsymbol{e}_{\widehat{u}}, \boldsymbol{\mu}\right\rangle_{\partial \mathcal{T}_{h}}=0, \\
& \left\langle\nu \mathrm{E}_{\mathrm{L}} \boldsymbol{n}-e_{p} \boldsymbol{n}-\boldsymbol{\alpha}\left(\boldsymbol{e}_{u}-\boldsymbol{e}_{\widehat{u}}\right), \boldsymbol{\mu}\right\rangle_{\partial \mathcal{T}_{h} \backslash \partial \Omega}=0, \\
& \left(e_{p}, 1\right)_{\mathcal{T}_{h}}=\left(\Pi_{P} p-p, 1\right)_{\mathcal{T}_{h}},
\end{aligned}
$$

for all $(\mathrm{G}, \boldsymbol{v}, q, \boldsymbol{\mu}) \in \mathrm{G}_{h} \times \boldsymbol{V}_{h} \times P_{h} \times \boldsymbol{M}_{h}$.

Proof. We begin by inserting the expression of the numerical trace given in (1.3g) into the second and the fifth equations (1.3b), (1.3e) defining the HDG method, then the numerical approximation $\left(\mathrm{L}_{h}, \boldsymbol{u}_{h}, p_{h}, \widehat{\boldsymbol{u}}_{h}\right)$ satisfies:

$$
\begin{aligned}
\left(\nu \mathrm{L}_{h}, \mathrm{G}\right)_{\mathcal{T}_{h}}+\left(\boldsymbol{u}_{h}, \nabla \cdot \mathrm{G}\right)_{\mathcal{T}_{h}}-\left\langle\widehat{\boldsymbol{u}}_{h}, \mathrm{G} \boldsymbol{n}\right\rangle_{\partial \mathcal{T}_{h}} & =0, \\
\left(\nu \mathrm{L}_{h}, \nabla \boldsymbol{v}\right)_{\mathcal{T}_{h}}-\left(p_{h}, \nabla \cdot \boldsymbol{v}\right)_{\mathcal{T}_{h}}-\left\langle\nu \mathrm{L}_{h} \boldsymbol{n}-p_{h} \boldsymbol{n}-\boldsymbol{\alpha}\left(\boldsymbol{u}_{h}-\widehat{\boldsymbol{u}}_{h}\right), \boldsymbol{v}\right\rangle_{\partial \mathcal{T}_{h}} & =(\boldsymbol{f}, \boldsymbol{v})_{\mathcal{T}_{h}}, \\
-\left(\boldsymbol{u}_{h}, \nabla q\right)_{\mathcal{T}_{h}}+\left\langle\widehat{\boldsymbol{u}}_{h} \cdot \boldsymbol{n}, q\right\rangle_{\partial \mathcal{T}_{h}} & =0, \\
\left\langle\widehat{\boldsymbol{u}}_{h}, \boldsymbol{\mu}\right\rangle_{\partial \Omega} & =\langle\boldsymbol{g}, \boldsymbol{\mu}\rangle_{\partial \Omega}, \\
\left\langle\mu \mathrm{L}_{h} \boldsymbol{n}-p_{h} \boldsymbol{n}-\boldsymbol{\alpha}\left(\boldsymbol{u}_{h}-\widehat{\boldsymbol{u}}_{h}\right), \boldsymbol{\mu}\right\rangle_{\partial \mathcal{T}_{h} \backslash \partial \Omega} & =0, \\
\left(p_{h}, 1\right)_{\mathcal{T}_{h}} & =0,
\end{aligned}
$$

for all $(\mathrm{G}, \boldsymbol{v}, q, \boldsymbol{\mu}) \in \mathrm{G}_{h} \times \boldsymbol{V}_{h} \times P_{h} \times \boldsymbol{M}_{h}$. Next, we note that the exact solution satisfies these same equations. Hence, by the Assumptions (A.1)-(A.7), we can equip the exact solution with the projection and obtain

$$
\begin{aligned}
(\nu \mathrm{L}, \mathrm{G})_{\mathcal{T}_{h}}+\left(\boldsymbol{\Pi}_{V} \boldsymbol{u}, \nabla \cdot \mathrm{G}\right)_{\mathcal{T}_{h}}-\left\langle\boldsymbol{\Pi}_{M} \boldsymbol{u}, \mathrm{G} \boldsymbol{n}\right\rangle_{\partial \mathcal{T}_{h}} & =0 \\
\left(\nu \Pi_{G} \mathrm{~L}, \nabla \boldsymbol{v}\right)_{\mathcal{T}_{h}}-\left(\Pi_{P} p, \nabla \cdot \boldsymbol{v}\right)_{\mathcal{T}_{h}}-\left\langle\nu \Pi_{G} \mathrm{~L} \boldsymbol{n}-\Pi_{P} p \boldsymbol{n}\right. & \left.-\boldsymbol{\alpha}\left(\boldsymbol{\Pi}_{V} \boldsymbol{u}-\boldsymbol{\Pi}_{M} \boldsymbol{u}\right), \boldsymbol{v}\right\rangle_{\partial \mathcal{T}_{h}} \\
& =(\boldsymbol{f}, \boldsymbol{v})_{\mathcal{T}_{h}}, \\
-\left(\boldsymbol{\Pi}_{V} \boldsymbol{u}, \nabla q\right)_{\mathcal{T}_{h}}+\left\langle\boldsymbol{\Pi}_{M} \boldsymbol{u} \cdot \boldsymbol{n}, q\right\rangle_{\partial \mathcal{T}_{h}} & =0, \\
\left\langle\boldsymbol{\Pi}_{M} \boldsymbol{u}, \boldsymbol{\mu}\right\rangle_{\partial \Omega} & =\langle\boldsymbol{g}, \boldsymbol{\mu}\rangle_{\partial \Omega}, \\
\left\langle\mu \Pi_{G} \mathrm{~L} \boldsymbol{n}-\Pi_{P} p \boldsymbol{n}-\boldsymbol{\alpha}\left(\boldsymbol{\Pi}_{V} \boldsymbol{u}-\boldsymbol{\Pi}_{M} \boldsymbol{u}\right), \boldsymbol{\mu}\right\rangle_{\partial \mathcal{T}_{h} \backslash \partial \Omega} & =0 \\
(p, 1)_{\mathcal{T}_{h}} & =0
\end{aligned}
$$


for all $(\mathrm{G}, \boldsymbol{v}, q, \boldsymbol{\mu}) \in \mathrm{G}_{h} \times \boldsymbol{V}_{h} \times P_{h} \times \boldsymbol{M}_{h}$. If we now subtract the first set of equations from this one, we obtain the result. This completes the proof of Lemma 5.1 .

Step 2: Estimate of the velocity gradient. We are now ready to obtain our first estimate by using a standard energy argument.

Proposition 5.2. We have

$$
\nu\left\|\mathrm{E}_{\mathrm{L}}\right\|_{\mathcal{T}_{h}}^{2}+\left\langle\boldsymbol{\alpha}\left(\boldsymbol{e}_{u}-\boldsymbol{e}_{\widehat{u}}\right), \boldsymbol{e}_{u}-\boldsymbol{e}_{\widehat{u}}\right\rangle_{\partial \mathcal{T}_{h}}=\nu\left(\Pi_{G} \mathrm{~L}-\mathrm{L}, \mathrm{E}_{\mathrm{L}}\right)_{\mathcal{T}_{h}} .
$$

Proof. If we take $\mathrm{G}=\nu \mathrm{E}_{\mathrm{L}}, \boldsymbol{v}=\boldsymbol{e}_{u}$ and $q=e_{p}$ in the first three error equations (5.1a) -(5.1c), respectively, and add them up. We obtain, after some algebraic manipulation,

where

$$
\nu\left\|\mathrm{E}_{\mathrm{L}}\right\|_{\mathcal{T}_{h}}^{2}+\Theta_{h}=\nu\left(\Pi_{G} \mathrm{~L}-\mathrm{L}, \mathrm{E}_{\mathrm{L}}\right)_{\mathcal{T}_{h}},
$$

$$
\Theta_{h}:=-\left\langle\boldsymbol{e}_{\widehat{u}}, \nu \mathrm{E}_{\mathrm{L}} \boldsymbol{n}\right\rangle_{\partial \mathcal{T}_{h}}+\left\langle\boldsymbol{\alpha}\left(\boldsymbol{e}_{\boldsymbol{u}}-\boldsymbol{e}_{\widehat{\boldsymbol{u}}}\right), \boldsymbol{e}_{u}\right\rangle_{\partial \mathcal{T}_{h}}+\left\langle\boldsymbol{e}_{\widehat{u}}, e_{p} \boldsymbol{n}\right\rangle_{\partial \mathcal{T}_{h}} .
$$

We thus obtain

$$
\begin{aligned}
\Theta_{h} & =\left\langle-\nu \mathrm{E}_{\mathrm{L}} \boldsymbol{n}+e_{p} \boldsymbol{n}, \boldsymbol{e}_{\widehat{u}}\right\rangle_{\partial \mathcal{T}_{h}}+\left\langle\boldsymbol{\alpha}\left(\boldsymbol{e}_{u}-\boldsymbol{e}_{\widehat{u}}\right), \boldsymbol{e}_{u}\right\rangle_{\partial \mathcal{T}_{h}} \\
& =\left\langle-\nu \mathrm{E}_{\mathrm{L}} \boldsymbol{n}+e_{p} \boldsymbol{n}+\boldsymbol{\alpha}\left(\boldsymbol{e}_{u}-\boldsymbol{e}_{\widehat{u}}\right), \boldsymbol{e}_{\widehat{u}}\right\rangle_{\partial \mathcal{T}_{h}}+\left\langle\boldsymbol{\alpha}\left(\boldsymbol{e}_{u}-\boldsymbol{e}_{\widehat{u}}\right), \boldsymbol{e}_{u}-\boldsymbol{e}_{\widehat{u}}\right\rangle_{\partial \mathcal{T}_{h}} \\
& =\left\langle\boldsymbol{\alpha}\left(\boldsymbol{e}_{u}-\boldsymbol{e}_{\widehat{u}}\right), \boldsymbol{e}_{u}-\boldsymbol{e}_{\widehat{u}}\right\rangle_{\partial \mathcal{T}_{h}},
\end{aligned}
$$

by the error equations (5.1d) and (5.1e). This completes the proof.

As a straightforward consequence of Proposition 5.2 and the Assumption (A.8), we obtain the first error estimate for the methods:

$$
\left\|\mathrm{E}_{\mathrm{L}}\right\|_{\mathcal{T}_{h}} \leq\left\|\Pi_{G} \mathrm{~L}-\mathrm{L}\right\|_{\mathcal{T}_{h}}
$$

This completes the proof of Theorem 2.1. Moreover, we also have the following estimate:

$$
\left\|\boldsymbol{e}_{u}-\boldsymbol{e}_{\widehat{u}}\right\|_{\boldsymbol{\alpha} / \nu} \leq\left\|\mathrm{L}-\Pi_{G} \mathrm{~L}\right\|_{\mathcal{T}_{h}} .
$$

Step 3: Estimate of the pressure. Next, we show how to use the previous result to obtain the estimate of the pressure.

Proposition 5.3. Let $\boldsymbol{P}: \boldsymbol{H}^{1}\left(\mathcal{T}_{h}\right) \rightarrow \boldsymbol{V}_{h}$ be any projection such that $(\boldsymbol{P} \boldsymbol{w}-$ $\boldsymbol{w}, \boldsymbol{v})_{\mathcal{T}_{h}}=0$ for all $\boldsymbol{v} \in \nabla P_{h}$. Then we have

$$
\left\|e_{p}-\overline{\left(p-\Pi_{P} p\right)}\right\|_{\mathcal{T}_{h}} \leq C C_{p, \alpha / \nu} \nu\left\|\mathrm{L}-\Pi_{G} \mathrm{~L}\right\|_{\mathcal{T}_{h}},
$$

where $C$ solely depends on the shape of the domain $\Omega$, and $C_{p, \alpha / \nu}$ is defined in Theorem 2.2 .

Proof. It is well known [3] that for any function $q \in L^{2}(\Omega)$ such that $(q, 1)_{\Omega}=0$ we have

$$
\|q\|_{\Omega} \leq \kappa \sup _{\boldsymbol{w} \in \boldsymbol{H}_{0}^{1}(\Omega) \backslash\{\mathbf{0}\}} \frac{(q, \nabla \cdot \boldsymbol{w})_{\Omega}}{\|\boldsymbol{w}\|_{\boldsymbol{H}^{1}(\Omega)}},
$$

for some constant $\kappa$ independent of $q$. By the last error equation ( $(\underline{5.1 \mathrm{f}})$, we see that we can apply the above result to $q:=e_{p}-\overline{e_{p}}$. Hence we have that

$$
\left\|e_{p}-\overline{e_{p}}\right\|_{\Omega} \leq \kappa \sup _{\boldsymbol{w} \in \boldsymbol{H}_{0}^{1}(\Omega) \backslash\{\mathbf{0}\}} \frac{\left(e_{p}-\overline{e_{p}}, \nabla \cdot \boldsymbol{w}\right)_{\Omega}}{\|\boldsymbol{w}\|_{\boldsymbol{H}^{1}(\Omega)}} .
$$


Next, we work on the numerator in the above expression. We have

$$
\left(e_{p}, \nabla \cdot \boldsymbol{w}\right)_{\Omega}=-\left(\nabla e_{p}, \boldsymbol{P} \boldsymbol{w}\right)_{\mathcal{T}_{h}}+\left\langle e_{p}, \boldsymbol{w} \cdot \boldsymbol{n}\right\rangle_{\partial \mathcal{T}_{h}} .
$$

By the second error equation (5.1b) with $\boldsymbol{v}:=\boldsymbol{P} \boldsymbol{w}$, we get that

$$
\begin{aligned}
\left(e_{p}, \nabla \cdot \boldsymbol{w}\right) & =-\left(\nabla \cdot\left(\nu \mathrm{E}_{L}\right), \boldsymbol{P} \boldsymbol{w}\right)_{\mathcal{T}_{h}}+\left\langle\boldsymbol{\alpha}\left(\boldsymbol{e}_{u}-\boldsymbol{e}_{\widehat{u}}\right), \boldsymbol{P} \boldsymbol{w}\right\rangle_{\partial \mathcal{T}_{h}}+\left\langle e_{p}, \boldsymbol{w} \cdot \boldsymbol{n}\right\rangle_{\partial \mathcal{T}_{h}} \\
& =\left(\nu \mathrm{E}_{L}, \nabla \boldsymbol{w}\right)_{\mathcal{T}_{h}}+\left\langle\boldsymbol{\alpha}\left(\boldsymbol{e}_{u}-\boldsymbol{e}_{\widehat{u}}\right), \boldsymbol{P} \boldsymbol{w}\right\rangle_{\partial \mathcal{T}_{h}}+\left\langle-\nu \mathrm{E}_{L} \boldsymbol{n}+e_{p} \boldsymbol{n}, \boldsymbol{\Pi}_{M} \boldsymbol{w}\right\rangle_{\partial \mathcal{T}_{h}} \\
& =\left(\nu \mathrm{E}_{L}, \nabla \boldsymbol{w}\right)_{\mathcal{T}_{h}}+\left\langle\boldsymbol{\alpha}\left(\boldsymbol{e}_{u}-\boldsymbol{e}_{\widehat{u}}\right), \boldsymbol{P} \boldsymbol{w}-\boldsymbol{\Pi}_{M} \boldsymbol{w}\right\rangle_{\partial \mathcal{T}_{h}},
\end{aligned}
$$

by the fifth error equation (5.1e) with $\mu=\boldsymbol{\Pi}_{M} \boldsymbol{w}$ and by the fact that $\boldsymbol{w} \in \boldsymbol{H}_{0}^{1}(\Omega)$.

By the Cauchy-Schwarz inequality, we get that

$$
\begin{aligned}
\left|\left(e_{p}, \nabla \cdot \boldsymbol{w}\right)\right| & \leq \nu\left\|\mathrm{E}_{L}\right\|_{\mathcal{T}_{h}}\|\nabla \boldsymbol{w}\|_{\Omega}+\left\|\boldsymbol{e}_{u}-\boldsymbol{e}_{\widehat{u}}\right\|_{\boldsymbol{\alpha} / \nu} \frac{\left\langle\boldsymbol{\alpha}\left(\boldsymbol{e}_{u}-\boldsymbol{e}_{\widehat{u}}\right), \boldsymbol{P} \boldsymbol{w}-\boldsymbol{\Pi}_{M} \boldsymbol{w}\right\rangle_{\partial \mathcal{T}_{h}}}{\left\|\boldsymbol{e}_{u}-\boldsymbol{e}_{\widehat{u}}\right\|_{\boldsymbol{\alpha} / \nu}} \\
& \leq \nu \mathbb{C}\left[\left\|\mathrm{E}_{L}\right\|_{\mathcal{T}_{h}}+\left\|\boldsymbol{e}_{u}-\boldsymbol{e}_{\widehat{u}}\right\|_{\boldsymbol{\alpha} / \nu}\right]\|\boldsymbol{w}\|_{\boldsymbol{H}^{1}(\Omega)}
\end{aligned}
$$

where

$$
\mathbb{C}:=\max \left\{1, \frac{\left\langle\boldsymbol{\alpha}\left(\boldsymbol{e}_{u}-\boldsymbol{e}_{\widehat{u}}\right) / \nu, \boldsymbol{P} \boldsymbol{w}-\boldsymbol{\Pi}_{M} \boldsymbol{w}\right\rangle_{\partial \mathcal{T}_{h}}}{\left\|\boldsymbol{e}_{u}-\boldsymbol{e}_{\widehat{u}}\right\|_{\boldsymbol{\alpha} / \nu}\|\boldsymbol{w}\|_{\boldsymbol{H}^{1}(\Omega)}}\right\} .
$$

Now, by the estimates (5.2),

$$
\left|\left(e_{p}, \nabla \cdot \boldsymbol{w}\right)\right| \leq \nu \mathbb{C}\left\|\mathrm{L}-\Pi_{G} \mathrm{~L}\right\|_{\mathcal{T}_{h}}\|\boldsymbol{w}\|_{\boldsymbol{H}^{1}(\Omega)} \leq \nu C_{p, \alpha / \nu}\left\|\mathrm{L}-\Pi_{G} \mathrm{~L}\right\|_{\mathcal{T}_{h}}\|\boldsymbol{w}\|_{\boldsymbol{H}^{1}(\Omega)},
$$

provided that $\boldsymbol{e}_{u}-\boldsymbol{e}_{\widehat{u}}$ lies in the auxiliary space $\mathcal{M}_{n, h}^{\perp}$ given by (2.1). We claim that this is indeed the case. To see that, note that for any face $F$ of the element $K \in \Omega_{h}$, we have that $\left.\left(\boldsymbol{e}_{u}-\boldsymbol{e}_{\widehat{u}}\right)\right|_{F} \in \boldsymbol{M}(F)$ by Assumption (A.6). Also, for any $q \in P(K)$ such that $(q, \nabla \cdot \boldsymbol{v})_{K}=0$ whenever $\boldsymbol{v} \in \boldsymbol{V}(K)$, we have that

$$
\left\langle\left(\boldsymbol{e}_{u}-\boldsymbol{e}_{\widehat{u}}\right) \cdot \boldsymbol{n}, q\right\rangle_{\partial K}=\left(\nabla \cdot \boldsymbol{e}_{u}, q\right)=0,
$$

by the error equation (5.1c). This proves the claim.

As a consequence, we obtain that

$$
\left\|e_{p}-\overline{e_{p}}\right\|_{\mathcal{T}_{h}} \leq C C_{p, \alpha / \nu} \nu\left\|\mathrm{L}-\Pi_{G} \mathrm{~L}\right\|_{\mathcal{T}_{h}} .
$$

Finally, the result follows from the fact that $\overline{e_{p}}=\overline{\Pi_{P} p-p}$ by the error equation (5.1f). This completes the proof of Proposition [5.3.

Theorem 2.2 follows directly from the above result.

Step 4: Estimate of the velocity. We are now ready to obtain a key identity for the projection of the error in the velocity by using a duality argument.

Lemma 5.4. We have

$$
\left(\boldsymbol{e}_{\boldsymbol{u}}, \boldsymbol{\theta}\right)_{\mathcal{T}_{h}}=\nu\left(\Pi_{G} \mathrm{~L}-\mathrm{L}, \mathrm{Z}\right)_{\mathcal{T}_{h}}+\nu\left(\mathrm{L}-\mathrm{L}_{h}, \mathrm{Z}-\Pi_{G}^{*} \mathrm{Z}\right)_{\mathcal{T}_{h}} .
$$

Proof. We have

$$
\left(\boldsymbol{e}_{u}, \boldsymbol{\theta}\right)_{\mathcal{T}_{h}}=\nu\left(\mathrm{E}_{L}, \mathrm{Z}-\nabla \boldsymbol{\sigma}\right)_{\mathcal{T}_{h}}+\left(\boldsymbol{e}_{u}, \nabla \cdot(\nu \mathrm{Z})-\nabla \eta\right)_{\mathcal{T}_{h}}+\left(e_{p}, \nabla \cdot \boldsymbol{\sigma}\right)_{\mathcal{T}_{h}},
$$


by the first three equations of the dual problem (2.2). Rearranging terms, we get

$$
\begin{aligned}
\left(\boldsymbol{e}_{u}, \boldsymbol{\theta}\right)_{\mathcal{T}_{h}} & =\left(\mathrm{E}_{L}, \nu \mathrm{Z}\right)_{\mathcal{T}_{h}}+\left(\boldsymbol{e}_{u}, \nabla \cdot(\nu \mathrm{Z})\right)_{\mathcal{T}_{h}} \\
& -\left(\nu \mathrm{E}_{L}, \nabla \boldsymbol{\sigma}\right)_{\mathcal{T}_{h}}+\left(e_{p}, \nabla \cdot \boldsymbol{\sigma}\right)_{\mathcal{T}_{h}} \\
& -\left(\boldsymbol{e}_{u}, \nabla \eta\right)_{\mathcal{T}_{h}} \\
& =\left(\mathrm{E}_{L}, \nu \Pi_{G}^{*} \mathrm{Z}\right)_{\mathcal{T}_{h}}+\left(\boldsymbol{e}_{u}, \nabla \cdot\left(\nu \Pi_{G}^{*} \mathrm{Z}\right)\right)_{\mathcal{T}_{h}} \\
& -\left(\nu \mathrm{E}_{L}, \nabla \boldsymbol{\sigma}\right)_{\mathcal{T}_{h}}+\left(e_{p}, \nabla \cdot \boldsymbol{\sigma}\right)_{\mathcal{T}_{h}} \\
& -\left(\boldsymbol{e}_{u}, \nabla \Pi_{P}^{*} \eta\right)_{\mathcal{T}_{h}} \\
& +\left(\mathrm{E}_{L}, \nu\left(\mathrm{Z}-\Pi_{G}^{*} \mathrm{Z}\right)\right)_{\mathcal{T}_{h}}+\left(\boldsymbol{e}_{u}, \nu \nabla \cdot\left(\mathrm{Z}-\Pi_{G}^{*} \mathrm{Z}\right)\right)_{\mathcal{T}_{h}}-\left(\boldsymbol{e}_{u}, \nabla\left(\eta-\Pi_{P}^{*} \eta\right)\right)_{\mathcal{T}_{h}},
\end{aligned}
$$

integrating by parts of the above equation and by the Assumption $A$, we obtain

$$
\begin{aligned}
\left(\boldsymbol{e}_{u}, \boldsymbol{\theta}\right)_{\mathcal{T}_{h}} & =\left(\mathrm{E}_{L}, \nu \Pi_{G}^{*} \mathrm{Z}\right)_{\mathcal{T}_{h}}+\left(\boldsymbol{e}_{u}, \nabla \cdot\left(\nu \Pi_{G}^{*} \mathrm{Z}\right)\right)_{\mathcal{T}_{h}} \\
& +\left(\nabla \cdot\left(\nu \mathrm{E}_{L}\right), \Pi_{V}^{*} \boldsymbol{\sigma}\right)_{\mathcal{T}_{h}}-\left(\nabla e_{p}, \Pi_{V}^{*} \boldsymbol{\sigma}\right)_{\mathcal{T}_{h}}-\left\langle\nu \mathrm{E}_{L} \boldsymbol{n}-e_{p} \boldsymbol{n}, \boldsymbol{\sigma}\right\rangle_{\partial \mathcal{T}_{h}} \\
& -\left(\boldsymbol{e}_{u}, \nabla \Pi_{P}^{*} \eta\right)_{\mathcal{T}_{h}} \\
& +\left(\mathrm{E}_{L}, \nu\left(\mathrm{Z}-\Pi_{G}^{*} \mathrm{Z}\right)\right)_{\mathcal{T}_{h}}+\left\langle\boldsymbol{e}_{u}, \nu\left(\mathrm{Z}-\Pi_{G}^{*} \mathrm{Z}\right) \boldsymbol{n}-\left(\eta-\Pi_{P}^{*} \eta\right) \boldsymbol{n}\right\rangle_{\partial \mathcal{T}_{h}},
\end{aligned}
$$

taking $\mathrm{G}=\nu \Pi_{G}^{*} \mathrm{Z}, \boldsymbol{v}=\boldsymbol{\Pi}_{V}^{*} \boldsymbol{\sigma}, q=\Pi_{P}^{*} \eta$ in the error equation (5.1a)-(5.1c) and inserting in the above identity, we get

$$
\left(\boldsymbol{e}_{u}, \boldsymbol{\theta}\right)_{\mathcal{T}_{h}}=\nu\left(\mathrm{E}_{L}, \mathrm{Z}-\Pi_{G}^{*} \mathrm{Z}\right)_{\mathcal{T}_{h}}+\nu\left(\Pi_{G} \mathrm{~L}-\mathrm{L}, \Pi_{G}^{*} \mathrm{Z}\right)_{\mathcal{T}_{h}}+\mathbb{T}_{1}+\mathbb{T}_{2},
$$

where

$$
\begin{aligned}
& \mathbb{T}_{1}:=\left\langle\boldsymbol{e}_{\widehat{u}}, \Pi_{G}^{*} \mathrm{Zn}\right\rangle_{\partial \mathcal{T}_{h}}-\left\langle\boldsymbol{e}_{\widehat{u}}, \Pi_{P}^{*} \eta \boldsymbol{n}\right\rangle_{\partial \mathcal{T}_{h}}+\left\langle\boldsymbol{e}_{u}, \nu\left(\mathrm{Z}-\Pi_{G}^{*} \mathrm{Z}\right) \boldsymbol{n}-\left(\eta-\Pi_{P}^{*} \eta\right) \boldsymbol{n}\right\rangle_{\partial \mathcal{T}_{h}}, \\
& \mathbb{T}_{2}:=\left\langle\boldsymbol{\alpha}\left(\boldsymbol{e}_{u}-\boldsymbol{e}_{\widehat{u}}\right), \Pi_{V}^{*} \boldsymbol{\sigma}\right\rangle_{\partial \mathcal{T}_{h}}-\left\langle\nu \mathrm{E}_{L} \boldsymbol{n}-e_{p} \boldsymbol{n}, \boldsymbol{\sigma}\right\rangle_{\partial \mathcal{T}_{h}} .
\end{aligned}
$$

Noting that $\left\langle\boldsymbol{e}_{\widehat{u}}, \mathrm{Zn}\right\rangle_{\partial \mathcal{T}_{h}}=\left\langle\boldsymbol{e}_{\widehat{u}}, \mathrm{Zn}\right\rangle_{\partial \Omega}=0$ and that $\left\langle\boldsymbol{e}_{\widehat{u}}, \eta \boldsymbol{n}\right\rangle_{\partial \mathcal{T}_{h}}=\left\langle\boldsymbol{e}_{\widehat{u}}, \eta \boldsymbol{n}\right\rangle_{\partial \Omega}=$ 0 , and inserting these two identities into $\mathbb{T}_{1}$, we obtain,

$$
\begin{aligned}
\mathbb{T}_{1} & :=-\left\langle\boldsymbol{e}_{\widehat{u}},\left(\mathrm{Z}-\Pi_{G}^{*} \mathrm{Z}\right) \boldsymbol{n}\right\rangle_{\partial \mathcal{T}_{h}}+\left\langle\boldsymbol{e}_{\widehat{u}},\left(\eta-\Pi_{P}^{*} \eta\right) \boldsymbol{n}\right\rangle_{\partial \mathcal{T}_{h}} \\
& +\left\langle\boldsymbol{e}_{u}, \nu\left(\mathrm{Z}-\Pi_{G}^{*} \mathrm{Z}\right) \boldsymbol{n}-\left(\eta-\Pi_{P}^{*} \eta\right) \boldsymbol{n}\right\rangle_{\partial \mathcal{T}_{h}} \\
& =\left\langle\boldsymbol{e}_{u}-\boldsymbol{e}_{\widehat{u}}, \nu\left(\mathrm{Z}-\Pi_{G}^{*} \mathrm{Z}\right) \boldsymbol{n}-\left(\eta-\Pi_{P}^{*} \eta\right) \boldsymbol{n}\right\rangle_{\partial \mathcal{T}_{h}} \\
& =\left\langle\boldsymbol{e}_{u}-\boldsymbol{e}_{\widehat{u}}, \boldsymbol{\alpha}^{*}\left(\boldsymbol{\Pi}_{M} \boldsymbol{\sigma}-\boldsymbol{\Pi}_{V}^{*} \boldsymbol{\sigma}\right)\right\rangle_{\partial \mathcal{T}_{h}} \\
& =\left\langle\boldsymbol{\alpha}\left(\boldsymbol{e}_{u}-\boldsymbol{e}_{\widehat{u}}\right), \boldsymbol{\Pi}_{M} \boldsymbol{\sigma}-\boldsymbol{\Pi}_{V}^{*} \boldsymbol{\sigma}\right\rangle_{\partial \mathcal{T}_{h}} .
\end{aligned}
$$

On the other hand, we have

$$
\begin{aligned}
\mathbb{T}_{2} & =\left\langle\boldsymbol{\alpha}\left(\boldsymbol{e}_{u}-\boldsymbol{e}_{\widehat{u}}\right), \boldsymbol{\Pi}_{V}^{*} \boldsymbol{\sigma}\right\rangle_{\partial \mathcal{T}_{h}}-\left\langle\nu \mathrm{E}_{L} \boldsymbol{n}-e_{p} \boldsymbol{n}, \boldsymbol{\Pi}_{M} \boldsymbol{\sigma}\right\rangle_{\partial \mathcal{T}_{h}} \\
& =\left\langle\boldsymbol{\alpha}\left(\boldsymbol{e}_{u}-\boldsymbol{e}_{\widehat{u}}\right), \boldsymbol{\Pi}_{V}^{*} \boldsymbol{\sigma}\right\rangle_{\partial \mathcal{T}_{h}}-\left\langle\boldsymbol{\alpha}\left(\boldsymbol{e}_{u}-\boldsymbol{e}_{\widehat{u}}\right), \boldsymbol{\Pi}_{M} \boldsymbol{\sigma}\right\rangle_{\partial \mathcal{T}_{h}},
\end{aligned}
$$

by the error equation (5.1e) and by the fact that $\left.\boldsymbol{\Pi}_{M} \boldsymbol{\sigma}\right|_{\partial \Omega}=0$ by the boundary condition for $\sigma(2.2 \mathrm{~d})$. Therefore, we get

$$
\mathbb{T}_{2}=-\left\langle\boldsymbol{\alpha}\left(e_{\boldsymbol{u}}-\boldsymbol{e}_{\widehat{\boldsymbol{u}}}\right), \boldsymbol{\Pi}_{V}^{*} \boldsymbol{\sigma}-\boldsymbol{\Pi}_{M} \boldsymbol{\sigma}\right\rangle_{\partial \mathcal{T}_{h}} .
$$

This implies that

$$
\begin{aligned}
\left(\boldsymbol{e}_{u}, \boldsymbol{\theta}\right)_{\mathcal{T}_{h}} & =\nu\left(\mathrm{E}_{L}, \mathrm{Z}-\Pi_{G}^{*} \mathrm{Z}\right)_{\mathcal{T}_{h}}+\nu\left(\Pi_{G} \mathrm{~L}-\mathrm{L}, \Pi_{G}^{*} \mathrm{Z}\right)_{\mathcal{T}_{h}} \\
& =\nu\left(\mathrm{E}_{L}, \mathrm{Z}-\Pi_{G}^{*} \mathrm{Z}\right)_{\mathcal{T}_{h}}-\nu\left(\Pi_{G} \mathrm{~L}-\mathrm{L}, \mathrm{Z}-\Pi_{G}^{*} \mathrm{Z}\right)_{\mathcal{T}_{h}}+\nu\left(\Pi_{G} \mathrm{~L}-\mathrm{L}, \mathrm{Z}\right)_{\mathcal{T}_{h}} \\
& =\nu\left(\Pi_{G} \mathrm{~L}-\mathrm{L}, \mathrm{Z}\right)_{\mathcal{T}_{h}}+\nu\left(\mathrm{L}-\mathrm{L}_{h}, \mathrm{Z}-\Pi_{G}^{*} \mathrm{Z}\right)_{\mathcal{T}_{h}} .
\end{aligned}
$$

This completes the proof. 
As a consequence, we immediately obtain an estimate on $\boldsymbol{e}_{u}$.

Corollary 5.5. If the regularity property (2.3) holds and Assumptions A, B are satisfied, then we have

$$
\left\|\boldsymbol{e}_{u}\right\|_{\mathcal{T}_{h}} \leq C \nu h\left\|\mathrm{~L}-\Pi_{G} \mathrm{~L}\right\|_{\mathcal{T}_{h}} .
$$

Proof. Taking $\boldsymbol{\theta}=\boldsymbol{e}_{u}$ in Lemma 5.4, we have

$$
\begin{aligned}
\left\|\boldsymbol{e}_{u}\right\|_{\mathcal{T}_{h}}^{2} & =\nu\left(\Pi_{G} \mathrm{~L}-\mathrm{L}, \mathrm{Z}\right)_{\mathcal{T}_{h}}+\nu\left(\mathrm{L}-\mathrm{L}_{h}, \mathrm{Z}-\Pi_{G}^{*} \mathrm{Z}\right)_{\mathcal{T}_{h}} \\
& =\nu\left(\Pi_{G} \mathrm{~L}-\mathrm{L}, \mathrm{Z}-\mathrm{Z}^{0}\right)_{\mathcal{T}_{h}}+\nu\left(\mathrm{L}-\mathrm{L}_{h}, \mathrm{Z}-\Pi_{G}^{*} \mathrm{Z}\right)_{\mathcal{T}_{h}},
\end{aligned}
$$

by Assumption (A.1) and Assumption (B.2), since $\left.\mathrm{Z}^{0}\right|_{K}$ is the average of $\mathrm{Z}$ on the element $K$ and hence belongs to $\mathrm{P}^{0}(K)$.

Now, after a simple application of the Cauchy-Schwarz inequality, we obtain

$$
\begin{aligned}
\left\|\boldsymbol{e}_{u}\right\|_{\mathcal{T}_{h}}^{2} & \leq C \nu h\left\|\mathrm{~L}-\Pi_{G} \mathrm{~L}\right\|_{\mathcal{T}_{h}}|\mathrm{Z}|_{1, \mathcal{T}_{h}}+\nu\left\|\mathrm{L}-\mathrm{L}_{h}\right\|_{\mathcal{T}_{h}}\left\|\mathrm{Z}-\Pi_{G}^{*} \mathrm{Z}\right\|_{\mathcal{T}_{h}} \\
& \leq C \nu h\left\|\mathrm{~L}-\Pi_{G} \mathrm{~L}\right\|_{\mathcal{T}_{h}}\left\|\boldsymbol{e}_{u}\right\|_{\mathcal{T}_{h}},
\end{aligned}
$$

by Assumption (B.1) and the regularity property (2.3). This completes the proof.

Next, we can obtain the following simple estimate.

Corollary 5.6. By the same assumption in Corollary 5.5, we have

$$
\left\|\boldsymbol{e}_{\widehat{u}}\right\|_{h} \leq C\left(h\left\|\mathrm{~L}-\Pi_{G} \mathrm{~L}\right\|_{\mathcal{T}_{h}}+\left\|\boldsymbol{e}_{u}\right\|_{\mathcal{T}_{h}}\right)
$$

Proof. From the first error equation (5.1a), we have that

$$
\left\langle\boldsymbol{e}_{\widehat{u}}, \mathrm{Gn}\right\rangle_{\partial K}=-\left(\Pi_{G} \mathrm{~L}-\mathrm{L}, \mathrm{G}\right)_{K}+\left(\mathrm{E}_{L}, \mathrm{G}\right)_{K}+\left(\boldsymbol{e}_{u}, \nabla \cdot \mathrm{G}\right)_{K},
$$

for all $\mathrm{G} \in \mathrm{G}(K)$. Hence, by a standard scaling argument (see [2]) we readily obtain that

$$
h_{K}^{\frac{1}{2}}\left\|\boldsymbol{e}_{\widehat{u}}\right\|_{\partial K} \leq C\left(h_{K}\left\|\mathrm{~L}-\Pi_{G} \mathrm{~L}\right\|_{K}+h_{K}\left\|\mathrm{E}_{L}\right\|_{K}+\left\|\boldsymbol{e}_{u}\right\|_{K}\right),
$$

and the estimate follows by using Theorem 2.1 .

Finally, Theorem 2.3 follows by the above two corollaries.

Step 5: Estimate of the postprocessed velocity. By the PoincaréFriedrichs inequality, we have that

$$
\left\|\boldsymbol{u}-\boldsymbol{u}_{h}^{*}\right\|_{K} \leq\left\|\overline{\boldsymbol{u}-\boldsymbol{u}_{h}^{*}}\right\|_{K}+C h_{K}\left\|\nabla\left(\boldsymbol{u}-\boldsymbol{u}_{h}^{*}\right)\right\|_{K},
$$

where $\bar{w}$ is the average of $w$ over $K$. But $\overline{\boldsymbol{u}_{h}^{*}}=\overline{\boldsymbol{u}_{h}}$, by the second equation defining $\boldsymbol{u}_{h}^{*},(1.4 \mathrm{~b})$, and $\overline{\boldsymbol{u}}=\overline{\boldsymbol{\Pi}_{V} \boldsymbol{u}}$ by Assumptions (A.2) and (C.1). This implies that

$$
\left\|\boldsymbol{u}-\boldsymbol{u}_{h}^{*}\right\|_{K} \leq\left\|\boldsymbol{\Pi}_{V} \boldsymbol{u}-\boldsymbol{u}_{h}\right\|_{K}+C h_{K}\left\|\nabla\left(\boldsymbol{u}-\boldsymbol{u}_{h}^{*}\right)\right\|_{K} .
$$

Now, for any $\boldsymbol{w} \in \boldsymbol{W}^{*}(K)$, we have that

$$
\begin{aligned}
\left\|\nabla\left(\boldsymbol{u}-\boldsymbol{u}_{h}^{*}\right)\right\|_{K}^{2} & =\left(\nabla\left(\boldsymbol{u}-\boldsymbol{u}_{h}^{*}\right), \nabla(\boldsymbol{u}-\boldsymbol{w})\right)_{K}+\left(\nabla\left(\boldsymbol{u}-\boldsymbol{u}_{h}^{*}\right), \nabla\left(\boldsymbol{w}-\boldsymbol{u}_{h}^{*}\right)\right)_{K} \\
& =\left(\nabla\left(\boldsymbol{u}-\boldsymbol{u}_{h}^{*}\right), \nabla(\boldsymbol{u}-\boldsymbol{w})\right)_{K}+\left(\mathrm{L}-\mathrm{L}_{h}, \nabla\left(\boldsymbol{w}-\boldsymbol{u}_{h}^{*}\right)\right)_{K},
\end{aligned}
$$


by the first equation defining the postprocessing $u_{h}^{*}$, 1.4a). Applying the CauchySchwarz inequality, we obtain that

$$
\begin{gathered}
\left\|\nabla\left(\boldsymbol{u}-\boldsymbol{u}_{h}^{*}\right)\right\|_{K}^{2} \leq\left\|\nabla\left(\boldsymbol{u}-\boldsymbol{u}_{h}^{*}\right)\right\|_{K}\|\nabla(\boldsymbol{u}-\boldsymbol{w})\|_{K}+\left\|\mathrm{L}-\mathrm{L}_{h}\right\|_{K}\left\|\nabla\left(\boldsymbol{w}-\boldsymbol{u}_{h}^{*}\right)\right\|_{K}, \\
\leq\left\|\nabla\left(\boldsymbol{u}-\boldsymbol{u}_{h}^{*}\right)\right\|_{K}\left(\|\nabla(\boldsymbol{u}-\boldsymbol{w})\|_{K}+\left\|\mathrm{L}-\mathrm{L}_{h}\right\|_{K}\right) \\
\quad+\left\|\mathrm{L}-\mathrm{L}_{h}\right\|_{K}\|\nabla(\boldsymbol{w}-\boldsymbol{u})\|_{K},
\end{gathered}
$$

and, after simple applications of Young's inequality and some algebraic manipulations, we get that

$$
\left\|\nabla\left(\boldsymbol{u}-\boldsymbol{u}_{h}^{*}\right)\right\|_{K}^{2} \leq 3\left(\left\|\mathrm{~L}-\mathrm{L}_{h}\right\|_{K}^{2}+\|\nabla(\boldsymbol{u}-\boldsymbol{w})\|_{K}^{2}\right) .
$$

This implies that

$$
\left\|\boldsymbol{u}-\boldsymbol{u}_{h}^{*}\right\|_{K} \leq\left\|\boldsymbol{\Pi}_{V} \boldsymbol{u}-\boldsymbol{u}_{h}\right\|_{K}+C h_{K}\left(\left\|\mathrm{~L}-\mathrm{L}_{h}\right\|_{K}+\|\nabla(\boldsymbol{u}-\boldsymbol{w})\|_{K}\right),
$$

and so,

$$
\left\|\boldsymbol{u}-\boldsymbol{u}_{h}^{*}\right\|_{\mathcal{T}_{h}} \leq\left\|\boldsymbol{\Pi}_{V} \boldsymbol{u}-\boldsymbol{u}_{h}\right\|_{\mathcal{T}_{h}}+C h\left(\left\|\mathrm{~L}-\mathrm{L}_{h}\right\|_{\mathcal{T}_{h}}+\|\nabla(\boldsymbol{u}-\boldsymbol{w})\|_{\mathcal{T}_{h}}\right) .
$$

This completes the proof of Theorem 2.4

\section{Concluding Remarks}

We have presented a technique for the a priori error analysis of HDG methods for Stokes equations and have shown how to use it to devise superconvergent methods from similar methods for the simple diffusion equation. Next, we discuss how to recover superconvergence when the space $\boldsymbol{V}(K)$ does not satisfy Assumption (B.2). We then sketch the extension of the method to isotropic elasticity.

Superconvergence when the local space $V(K)$ is too small. Let us consider the method listed in Table 1 as $\mathbf{R T}_{\mathbf{k}}$, or the one listed in Table 3 as $\mathbf{R T}_{[\mathbf{k}]}$, for $k=0$. In this cases, the Assumption (B.2) is not satisfied because $\boldsymbol{V}(K)=\boldsymbol{P}_{0}(K)$. However, we can still obtain superconvergence of the projection of the error in the velocity as we show next.

Then, by the identity of Lemma 5.4 .

$$
\begin{array}{rlr}
\left(\boldsymbol{e}_{\boldsymbol{u}}, \boldsymbol{\theta}\right)_{\mathcal{T}_{h}} & =\nu\left(\Pi_{G} \mathrm{~L}-\mathrm{L}, \mathrm{Z}\right)_{\mathcal{T}_{h}}+\nu\left(\mathrm{L}-\mathrm{L}_{h}, \mathrm{Z}-\Pi_{G}^{*} \mathrm{Z}\right)_{\mathcal{T}_{h}} & \\
& =\nu\left(\Pi_{G} \mathrm{~L}-\mathrm{L}, \nabla \boldsymbol{\sigma}\right)_{\mathcal{T}_{h}}+\nu\left(\mathrm{L}-\mathrm{L}_{h}, \mathrm{Z}-\Pi_{G}^{*} \mathrm{Z}\right)_{\mathcal{T}_{h}} & \text { by (2.2a), } \\
& =-\nu\left(\nabla \cdot\left(\Pi_{G} \mathrm{~L}-\mathrm{L}\right), \boldsymbol{\sigma}\right)_{\mathcal{T}_{h}}+\nu\left(\mathrm{L}-\mathrm{L}_{h}, \mathrm{Z}-\Pi_{G}^{*} \mathrm{Z}\right)_{\mathcal{T}_{h}} & \text { by (2.2d) } \\
& =-\nu(\overline{\nabla \cdot \mathrm{L}}-\nabla \cdot \mathrm{L}, \boldsymbol{\sigma}-\overline{\boldsymbol{\sigma}})_{\mathcal{T}_{h}}+\nu\left(\mathrm{L}-\mathrm{L}_{h}, \mathrm{Z}-\Pi_{G}^{*} \mathrm{Z}\right)_{\mathcal{T}_{h}}, &
\end{array}
$$

and, proceeding as before, we can get that

$$
\left\|\boldsymbol{e}_{\boldsymbol{u}}\right\|_{\mathcal{T}_{h}} \leq C h^{2}\|\nabla \cdot \mathrm{L}\|_{\boldsymbol{H}^{1}(\Omega)}+C h\left\|\mathrm{~L}-\Pi_{G} \mathrm{~L}\right\|_{\mathcal{T}_{h}},
$$

and we obtain the wanted superconvergence. As a consequence, the postprocessing $\boldsymbol{u}_{h}^{*}$ also satisfies the estimate of Theorem 2.4 and hence converges with order two whenever the solution is smooth enough. These results complement the error estimates obtained in 9,10 . 
Isotropic elasticity. We end the paper by sketching the extension of our approach to the analysis of HDG methods for isotropic linear elasticity equations; see 14. The governing equations of linear elasticity for isotropic materials can be written as follows:

$$
\begin{aligned}
\mathrm{L}-\nabla \boldsymbol{u} & =0, & & \text { on } \Omega, \\
-\nabla \cdot(\mu \mathrm{L})+\nabla p & =0, & & \text { on } \Omega, \\
\epsilon p+\nabla \cdot \boldsymbol{u} & =0, & & \text { on } \Omega, \\
\boldsymbol{u} & =\boldsymbol{g}, & & \text { on } \partial \Omega,
\end{aligned}
$$

where $\epsilon=(1-2 \nu)(1+\nu) / E$. Here $E$ is Young's modulus and $\nu \in(0,1 / 2]$ is Poisson's ratio. The advantage of this formulation is that it holds for both compressible $(\nu \in(0,1 / 2))$ and incompressible $(\nu=1 / 2)$ materials. Clearly, for incompressible materials, this system of equations is nothing but the Stokes system (1.1).

For the case of compressible materials $(\epsilon>0)$, the weak formulation of the system is similar to the Stokes equation (1.3). In fact, the only difference is that we replace the equation (1.3c) by

$$
\left(\epsilon p_{h}, q\right)_{\mathcal{T}_{h}}-\left(\boldsymbol{u}_{h}, \nabla q\right)_{\mathcal{T}_{h}}+\left\langle\widehat{\boldsymbol{u}}_{h} \cdot \boldsymbol{n}, q\right\rangle_{\partial \mathcal{T}_{h}}=0 .
$$

Next, we argue that this difference does not affect the application of our approach to these equations.

Indeed, under the same Assumption A, we can obtain the following identity:

$$
\epsilon\left\|e_{p}\right\|_{\mathcal{T}_{h}}^{2}+\nu\left\|\mathrm{E}_{\mathrm{L}}\right\|_{\mathcal{T}_{h}}^{2}+\left\langle\boldsymbol{\alpha}\left(\boldsymbol{e}_{\boldsymbol{u}}-\boldsymbol{e}_{\widehat{\boldsymbol{u}}}\right), \boldsymbol{e}_{\boldsymbol{u}}-\boldsymbol{e}_{\widehat{\boldsymbol{u}}}\right\rangle_{\partial \mathcal{T}_{h}}=\nu\left(\Pi_{G} \mathrm{~L}-\mathrm{L}, \mathrm{E}_{\mathrm{L}}\right)_{\mathcal{T}_{h}} .
$$

Since $\epsilon>0$ and $\boldsymbol{\alpha}$ is semi-positive definite, we still have the estimate

$$
\left\|\mathrm{L}-\mathrm{L}_{h}\right\|_{\mathcal{T}_{h}} \leq 2\left\|\Pi_{G} \mathrm{~L}-\mathrm{L}\right\|_{\mathcal{T}_{h}} .
$$

To get the estimate of the pressure, we can use the same argument (Proposition 5.3) since we only need the error equation (5.1b).

Finally, if we modify the dual problem (2.2) by inserting $-\epsilon \eta$ on the left-hand side of (2.2c), then, by using a similar duality argument, we obtain that

$$
\left(\boldsymbol{e}_{\boldsymbol{u}}, \boldsymbol{\theta}\right)_{\mathcal{T}_{h}}=\nu\left(\Pi_{G} \mathrm{~L}-\mathrm{L}, \mathrm{Z}\right)_{\mathcal{T}_{h}}+\nu\left(\mathrm{L}-\mathrm{L}_{h}, \mathrm{Z} \Pi_{G}^{*} \mathrm{Z}\right)_{\mathcal{T}_{h}}+\epsilon\left(e_{p}, \eta-\Pi_{P}^{*} \eta\right)_{\mathcal{T}_{h}} .
$$

Finally, assuming the same regularity property, and under the same Assumption B, we get

$$
\left\|\boldsymbol{\Pi}_{V} \boldsymbol{u}-\boldsymbol{u}_{h}\right\|_{\mathcal{T}_{h}} \leq C h\left\|\mathrm{~L}-\Pi_{G} \mathrm{~L}\right\|_{\mathcal{T}_{h}} .
$$

Clearly, the very same local postprocessing can be applied if Assumption $C$ is satisfied.

In conclusion, our projection-based analysis can be naturally extended to isotropic elasticity problems. The extension of this approach to methods for linear elasticity, which are based on weak symmetry formulations, is the subject of ongoing research.

\section{REFERENCES}

[1] F. Brezzi, J. Douglas, Jr., M. Fortin, and L. D. Marini, Efficient rectangular mixed finite element methods in two and three space variables, RAIRO Modél. Math. Anal. Numér. 21 (1987), 581-604. MR921828 (88j:65249)

[2] F. Brezzi, J. Douglas, Jr., and L. D. Marini, Two families of mixed finite elements for second order elliptic problems, Numer. Math. 47 (1985), 217-235. MR799685 (87g:65133)

[3] F. Brezzi and M. Fortin, Mixed and hybrid finite element methods, Springer-Verlag, 1991. MR1115205 (92d:65187) 
[4] B. Cockburn, J. Gopalakrishnan, N.C. Nguyen, J. Peraire, and F.J. Sayas, Analysis of an HDG method for Stokes flow, Math. Comp. 80 (2010), 723-760. MR2772094

[5] B. Cockburn, J. Gopalakrishnan, and F.-J. Sayas, A projection-based error analysis of HDG methods, Math. Comp. 79 (2010), 1351-1367. MR2629996(2011d:65354)

[6] B. Cockburn and F.J. Sayas, Divergence-conforming HDG methods for Stokes flow. Submitted.

[7] B. Cockburn, W. Qiu and K. Shi, Conditions for superconvergence of HDG methods for second-order elliptic problems, Math. Comp. 81 (2012), 1327-1353. MR2904581

[8] M. Dauge, Stationary Stokes and Navier-Stokes systems on two- or three-dimensional domains with corners. I. Linearized equations, SIAM J. Math. Anal. 20 (1989), no. 1, 74-97. MR977489 (90b:35191)

[9] M. Farhloul and M. Fortin, A new mixed finite element for the Stokes and elasticity problems, SIAM J. Numer. Anal. 30 (1993), no. 4, 971-990. MR1231323 (94g:65123)

[10] M. Farhloul and M. Fortin, A mixed finite element for the Stokes problem using quadrilateral elements, Adv. Comput. Math. 3 (1995), no. 1-2, 101-113. MR1314904 (96e:65068)

[11] L. Gastaldi and R.H. Nochetto, Sharp maximum norm error estimates for general mixed finite element approximations to second order elliptic equations, RAIRO Modél. Math. Anal. Numér. 23 (1989), 103-128. MR1015921 (91b:65125)

[12] R. B. Kellogg and J. E. Osborn, A regularity result for the Stokes problem in a convex polygon, J. Functional Analysis 21 (1976), no. 4, 397-431. MR0404849 (53:8649)

[13] J.-C. Nédélec, Mixed finite elements in $\mathbf{R}^{3}$, Numer. Math. 35 (1980), 315-341. MR.592160 (81k:65125)

[14] N.C. Nguyen and J. Peraire, Hybridizable discontinuous Galerkin methods for partial differential equations in continuum mechanics. J. Comp. Phy. 231 (2012), 5955-5988.

[15] N.C. Nguyen, J. Peraire, and B. Cockburn, A hybridizable discontinuous Galerkin method for Stokes flow, Comput. Methods Appl. Mech. Engrg. 199 (2010), 582-597. MR2796169 (2011m:65286)

[16] P. A. Raviart and J. M. Thomas, A mixed finite element method for second order elliptic problems, Mathematical Aspects of Finite Element Method, Lecture Notes in Math. 606 (I. Galligani and E. Magenes, eds.), Springer-Verlag, New York, 1977, pp. 292-315. MR0483555 (58:3547)

[17] R. Stenberg, A family of mixed finite elements for the elasticity problem, Numer. Math. 53 (1988), 513-538. MR954768 (89h:65192)

[18] R. Stenberg, Postprocessing schemes for some mixed finite elements, RAIRO Modél. Math. Anal. Numér. 25 (1991), 151-167. MR1086845 (92a:65303)

School of Mathematics, University of Minnesota, Minneapolis, Minnesota 55455

E-mail address: cockburn@math.umn.edu

School of Mathematics, University of Minnesota, Minneapolis, Minnesota 55455

E-mail address: shixx075@math.umn.edu 